\title{
Regularity of Commutators of Maximal Operators with Lipschitz Symbols
}

\author{
Feng Liu* and Guoru Wang
}

\begin{abstract}
This paper is devoted to studying Sobolev regularity properties of commutators of Hardy-Littlewood maximal operator and its fractional case with Lipschitz symbols, both in the global and local case. Some new pointwise estimates for the weak gradients of the above commutators will be established. As applications, some bounds for the above commutators on the Sobolev spaces will be obtained.
\end{abstract}

\section{Introduction}

\subsection{Background}

The regularity theory of maximal operators is an active topic of current research. A driving question related to this theory is whether a given maximal operator improves, preserves or destroys the a priori regularity of an initial datum $f$. In 1997, Kinnunen 16 first studied the Sobolev regularity for the Hardy-Littlewood maximal operator

$$
M f(x)=\sup _{r>0} \frac{1}{|B(x, r)|} \int_{B(x, r)}|f(y)| d y,
$$

where $B(x, r)$ is the open ball in $\mathbb{R}^{n}$ centered at $x$ with radius $r$ and $|B(x, r)|$ denotes its volume, and showed that $M$ is bounded on the first order Sobolev spaces $W^{1, p}\left(\mathbb{R}^{n}\right)$ for $1<p \leq \infty$, where

$$
W^{1, p}\left(\mathbb{R}^{n}\right):=\left\{f: \mathbb{R}^{n} \rightarrow \mathbb{R} \mid\|f\|_{1, p}:=\|f\|_{p}+\|\nabla f\|_{p}<\infty\right\}
$$

where $\|f\|_{W^{1, p}\left(\mathbb{R}^{n}\right)}:=\|f\|_{1, p},\|f\|_{p}:=\|f\|_{L^{p}\left(\mathbb{R}^{n}\right)}$ and $\nabla f=\left(D_{1} f, \ldots, D_{n} f\right)$ is the weak gradient of $f$. Later on, more and more scholars devoted to extending Kinnunen's result to various variants (see $7,17,19,23$ ). Since the derivative of maximal function has no sublinearity, the continuity of $M: W^{1, p}\left(\mathbb{R}^{n}\right) \rightarrow W^{1, p}\left(\mathbb{R}^{n}\right)$ for $1<p<\infty$ is affirmatively a nontrivial issue. This question was first answered by Luiro [27] and later extended to the local case in [28] and to the multilinear fractional case in [22]. The endpoint regularity

Received October 27, 2020; Accepted March 2, 2021.

Communicated by Sanghyuk Lee.

2020 Mathematics Subject Classification. 42B25, 46E35.

Key words and phrases. commutator, Hardy-Littlewood maximal operator, fractional variant, local version, Sobolev space.

*Corresponding author. 
properties of maximal operators can be found in [2,5,6, 8, 29. Other interesting works of regularity theory are $20,21,24,28$ for the boundedness for maximal operator on the fractional Sobolev spaces, Triebel-Lizorkin spaces and Besov spaces as well as [25,26] for the regularity properties for the commutators of maximal operators.

Recently, Liu, Xue and Zhang [26] studied the regularity properties for the commutators of Hardy-Littlewood maximal function. To be more precise, let $b$ be a locally integral function defined on $\mathbb{R}^{n}$, the commutators of the Hardy-Littlewood maximal operator is defined by

$$
[b, M](f)(x)=b(x) M f(x)-M(b f)(x), \quad x \in \mathbb{R}^{n} .
$$

The maximal commutator of $M$ with $b$ is defined as

$$
\mathfrak{M}_{b} f(x)=\sup _{r>0} \frac{1}{|B(x, r)|} \int_{B(x, r)}|b(x)-b(y)||f(y)| d y, \quad x \in \mathbb{R}^{n} .
$$

Liu, Xue and Zhang [26] established the following result.

Theorem 1.1. 26] Let $1<p_{1}, p_{2}, p<\infty$ and $1 / p=1 / p_{1}+1 / p_{2}$. If $b \in W^{1, p_{2}}\left(\mathbb{R}^{n}\right)$, then the map $[b, M]: W^{1, p_{1}}\left(\mathbb{R}^{n}\right) \rightarrow W^{1, p}\left(\mathbb{R}^{n}\right)$ is bounded and continuous. Moreover, if $f \in W^{1, p_{1}}\left(\mathbb{R}^{n}\right)$, then

$$
\|[b, M](f)\|_{1, p} \leq C_{n, p_{1}, p_{2}}\|b\|_{1, p_{2}}\|f\|_{1, p_{1}} .
$$

The above boundedness also holds for $\mathfrak{M}_{b}$.

Very recently, Liu and $\mathrm{Xi}$ [25] extended Theorem 1.1 to the fractional case. Let us introduce the commutators of fractional maximal operator.

Definition 1.2. Let $0 \leq \alpha<n$ and $M_{\alpha}$ be the fractional maximal operator on $\mathbb{R}^{n}$, i.e., for $f \in L_{\text {loc }}^{1}\left(\mathbb{R}^{n}\right)$,

$$
M_{\alpha} f(x)=\sup _{r>0} \frac{r^{\alpha}}{|B(x, r)|} \int_{B(x, r)}|f(y)| d y, \quad x \in \mathbb{R}^{n} .
$$

For a locally integral function $b$ defined on $\mathbb{R}^{n}$, the commutator of fractional maximal operator $M_{\alpha}$ with $b$ is defined by

$$
\left[b, M_{\alpha}\right](f)(x)=b(x) M_{\alpha} f(x)-M_{\alpha}(b f)(x), \quad x \in \mathbb{R}^{n} .
$$

The fractional maximal commutator of $M_{\alpha}$ with $b$ is defined as

$$
\mathfrak{M}_{b, \alpha} f(x)=\sup _{r>0} \frac{r^{\alpha}}{|B(x, r)|} \int_{B(x, r)}|b(x)-b(y)||f(y)| d y, \quad x \in \mathbb{R}^{n} .
$$

Clearly, $M_{\alpha}=M,\left[b, M_{\alpha}\right]=[b, M]$ and $\mathfrak{M}_{b, \alpha}=\mathfrak{M}_{b}$ when $\alpha=0$.

We now introduce the main results of [25] as follows. 
Theorem 1.3. [25] Let $1<p_{1}, p_{2}, p, p_{1} p_{2} /\left(p_{1}+p_{2}\right)<\infty, 0 \leq \alpha<n / p_{1}$ and $1 / q=$ $1 / p_{1}+1 / p_{2}-\alpha / n$. If $b \in W^{1, p_{2}}\left(\mathbb{R}^{n}\right)$, then the map $\left[b, M_{\alpha}\right]: W^{1, p_{1}}\left(\mathbb{R}^{n}\right) \rightarrow W^{1, q}\left(\mathbb{R}^{n}\right)$ is bounded. In particular, if $f \in W^{1, p_{1}}\left(\mathbb{R}^{n}\right)$, then

$$
\left|D_{i}\left[b, M_{\alpha}\right](f)(x)\right| \leq|b(x)| M_{\alpha}\left(D_{i} f\right)(x)+M_{\alpha}\left(b D_{i} f\right)(x)+\left|D_{i} b(x)\right| M_{\alpha} f(x)+M_{\alpha}\left(D_{i} b f\right)(x)
$$

for almost every $x \in \mathbb{R}^{n}$ and $i=1,2, \ldots, n$. Moreover,

$$
\left\|\left[b, M_{\alpha}\right](f)\right\|_{1, q} \leq C_{\alpha, n, p_{1}, p_{2}}\|b\|_{1, p_{2}}\|f\|_{1, p_{1}} .
$$

The same conclusions hold for $\mathfrak{M}_{b, \alpha}$.

In this paper we continue to focus on the Sobolev regularity properties for the commutators of Hardy-Littlewood maximal operator and its fractional version. More precisely, we shall establish some new results on the Sobolev regularity properties of the above commutators with Lipschitz symbols, both in the global and local case.

\subsection{The global case}

In 1990, Milman and Schonbek [30] first introduced the commutator of maximal operator and established the $L^{p}(1<p<\infty)$ bounds of $\left[b, M_{\mathcal{C}}\right]$ when $b \geq 0$ and $b \in \operatorname{BMO}\left(\mathbb{R}^{n}\right)$. Here $M_{\mathcal{C}}$ is the Hardy-Littlewood maximal operator associated to cubes. Subsequently, Bastero, Milman and Ruiz [3] improved the above result by removing the restrictive condition $b \geq 0$. It was shown in $\left[4\right.$ that the operator $\left[b, \widetilde{M}_{\mathcal{C}}\right]$ can be used in studying the product of a function in $H^{1}\left(\mathbb{R}^{n}\right)$ and a function in $\operatorname{BMO}\left(\mathbb{R}^{n}\right)$. Later on, the $L^{p} \rightarrow L^{q}$ bounds for the commutators of fractional maximal operator have been studied by many authors (see $9,12,32$ ). The maximal commutator was first studied by García-Cuerva, Harboure, Segovia and Torrea [10] who showed that the maximal commutator of $M_{\mathcal{C}}$ with $b$ is bounded on $L^{p}\left(\mathbb{R}^{n}\right)$ for $1<p<\infty$ if and only if $b \in \operatorname{BMO}\left(\mathbb{R}^{n}\right)$. Other interesting papers related to this topic can be consulted [1,31, among others. It should be pointed out that the corresponding results also hold for $[b, M],\left[b, M_{\alpha}\right], \mathfrak{M}_{b}$ or $\mathfrak{M}_{b, \alpha}$, which is based on the fact that the Hardy-Littlewood maximal operator associated to balls has same properties as the Hardy-Littlewood maximal operator associated to cubes.

From Theorems 1.1 and 1.3 , one sees that, the assumptions that these symbols $b$ belong to Sobolev spaces guarantee certain Sobolev regularity for the commutators of the Hardy-Littlewood maximal operator and its fractional version. A natural question is the following

Question 1.4. Do the commutators $[b, M],\left[b, M_{\alpha}\right], \mathfrak{M}_{b}$ and $\mathfrak{M}_{b, \alpha}$ have somewhat Sobolev regularity properties when the symbols $b$ are not Sobolev functions? 
Question 1.4 is one of main motivations in this paper. In order to formulate our main results, let us introduce Lipschitz space.

Definition 1.5 (Lipschitz space). The homogeneous Lipschitz space $\operatorname{Lip}\left(\mathbb{R}^{n}\right)$ is defined by

$$
\operatorname{Lip}\left(\mathbb{R}^{n}\right):=\left\{f: \mathbb{R}^{n} \rightarrow \mathbb{C} \text { continuous : }\|f\|_{\operatorname{Lip}\left(\mathbb{R}^{n}\right)}<\infty\right\}
$$

where

$$
\|f\|_{\operatorname{Lip}\left(\mathbb{R}^{n}\right)}:=\sup _{x \in \mathbb{R}^{n}} \sup _{h \in \mathbb{R}^{n} \backslash\{0\}} \frac{|f(x+h)-f(x)|}{|h|}<\infty .
$$

The inhomogeneous Lipschitz space $\operatorname{Lip}\left(\mathbb{R}^{n}\right)$ is given by

$$
\operatorname{Lip}\left(\mathbb{R}^{n}\right):=\left\{f: \mathbb{R}^{n} \rightarrow \mathbb{C} \text { continuous }:\|f\|_{\operatorname{Lip}\left(\mathbb{R}^{n}\right)}<\infty\right\}
$$

where

$$
\|f\|_{\operatorname{Lip}\left(\mathbb{R}^{n}\right)}:=\|f\|_{\infty}+\|f\|_{\operatorname{Lip}\left(\mathbb{R}^{n}\right)}<\infty .
$$

Remark 1.6. Let $b \in \operatorname{Lip}\left(\mathbb{R}^{n}\right)$. Then the weak partial derivatives $D_{i} b, i=1, \ldots, n$, exist almost everywhere. Moreover, it holds that

$$
D_{i} b(x)=\lim _{h \rightarrow 0} \frac{b\left(x+h e_{i}\right)-b(x)}{h}
$$

and

$$
\left|D_{i} b(x)\right| \leq\|b\|_{\operatorname{Lip}\left(\mathbb{R}^{n}\right)}
$$

for almost every $x \in \mathbb{R}^{n}$. Here $e_{l}=(0, \ldots, 0, i, 0, \ldots, 0)$ is the canonical $i$-th base vector in $\mathbb{R}^{n}$ for $i=1, \ldots, n$.

To see (1.1) and 1.2), let us fix $i=1, \ldots, n$. Since $b$ is Lipschitz continuous, then by Rademacher's theorem, we know that $b$ is differentiable almost everywhere. Then the partial derivatives $D_{i} b$ exists almost everywhere and 1.1 holds. For almost every $x \in \mathbb{R}^{n}$, we get by 1.1 that

$$
\left|D_{i} b(x)\right|=\left|\lim _{h \rightarrow 0} \frac{b\left(x+h e_{i}\right)-b(x)}{h}\right| \leq \lim _{h \rightarrow 0} \frac{\left|b\left(x+h e_{i}\right)-b(x)\right|}{|h|} \leq\|b\|_{\operatorname{Lip}\left(\mathbb{R}^{n}\right)},
$$

which gives 1.2 . It follows from that

$$
|\nabla b(x)| \leq \sqrt{n}\|b\|_{\operatorname{Lip}\left(\mathbb{R}^{n}\right)}
$$

for almost every $x \in \mathbb{R}^{n}$.

We also list some comments on $\left[b, M_{\alpha}\right]$ and $\mathfrak{M}_{b, \alpha}$, which are useful for our aim. Remark 1.7. Let $1<p<\infty, 0 \leq \alpha<n / p$ and $1 / q=1 / p-\alpha / n$. If $b \in L^{\infty}\left(\mathbb{R}^{n}\right)$, then the following are valid: 
(i) If $f \in L^{p}\left(\mathbb{R}^{n}\right)$, then

$$
\left\|M_{\alpha} f\right\|_{q} \leq A_{\alpha, n, p}\|f\|_{p}
$$

where $A_{\alpha, n, p}:=\left\|M_{\alpha}\right\|_{L^{p}\left(\mathbb{R}^{n}\right) \rightarrow L^{q}\left(\mathbb{R}^{n}\right)}$. By (1.4) and the sublinearity of $M_{\alpha}$, we see that $M_{\alpha}: L^{p}\left(\mathbb{R}^{n}\right) \rightarrow L^{q}\left(\mathbb{R}^{n}\right)$ is continuous.

(ii) The operator $\left[b, M_{\alpha}\right]$ is neither positive nor sublinear. Applying Hölder's inequality and (1.4), one has

$$
\left\|\left[b, M_{\alpha}\right](f)\right\|_{q} \leq 2 A_{\alpha, n, p}\|b\|_{\infty}\|f\|_{p}
$$

On the other hand, it is easy to see that

$$
\left|\left[b, M_{\alpha}\right](f)-\left[b, M_{\alpha}\right](g)\right| \leq|b| M_{\alpha}(f-g)+M_{\alpha}(b(f-g)),
$$

which together with (1.4) implies that the map $\left[b, M_{\alpha}\right]: L^{p}\left(\mathbb{R}^{n}\right) \rightarrow L^{q}\left(\mathbb{R}^{n}\right)$ is continuous.

(iii) The operator $\mathfrak{M}_{b, \alpha}$ is positive and sublinear. Observe that

$$
\mathfrak{M}_{b, \alpha} f(x) \leq|b(x)| M_{\alpha} f(x)+M_{\alpha}(b f)(x), \quad x \in \mathbb{R}^{n} .
$$

Inequality (1.6) together with Hölder's inequality and $(1.4)$ implies that

$$
\left\|\mathfrak{M}_{b, \alpha} f\right\|_{q} \leq 2 A_{\alpha, n, p}\|b\|_{\infty}\|f\|_{p} .
$$

Moreover, by the sublinearity and boundedness for $\mathfrak{M}_{b, \alpha}$, we see that the map $\mathfrak{M}_{b, \alpha}: L^{p}\left(\mathbb{R}^{n}\right) \rightarrow L^{q}\left(\mathbb{R}^{n}\right)$ is continuous.

Now we shall provide a positive answer to Question 1.4 by the following theorem.

Theorem 1.8. Let $1<p<\infty, 0 \leq \alpha<n / p$ and $1 / q=1 / p-\alpha / n$. If $b \in \operatorname{Lip}\left(\mathbb{R}^{n}\right)$, then the map $\left[b, M_{\alpha}\right]: W^{1, p}\left(\mathbb{R}^{n}\right) \rightarrow W^{1, q}\left(\mathbb{R}^{n}\right)$ is bounded and continuous. In particular, if $f \in W^{1, p}\left(\mathbb{R}^{n}\right)$, then for each $i \in\{1, \ldots, n\}$ and almost every $x \in \mathbb{R}^{n}$,

$$
\left|\nabla\left(\left[b, M_{\alpha}\right](f)\right)(x)\right| \leq 2 \sqrt{n}\|b\|_{L i p\left(\mathbb{R}^{n}\right)} M_{\alpha} f(x)+2\|b\|_{\infty} M_{\alpha}|\nabla f|(x) .
$$

Consequently,

$$
\left\|\left[b, M_{\alpha}\right](f)\right\|_{1, q} \leq 2 A_{\alpha, n, p}(n+1)\|b\|_{\operatorname{Lip}\left(\mathbb{R}^{n}\right)}\|f\|_{1, p},
$$

where $A_{\alpha, n, p}$ is given as in (1.4). Inequalities (1.8) and (1.9) also hold for $\mathfrak{M}_{b, \alpha}$.

Remark 1.9. It is unknown that whether the map $\mathfrak{M}_{b, \alpha}: W^{1, p}\left(\mathbb{R}^{n}\right) \rightarrow W^{1, q}\left(\mathbb{R}^{n}\right)$ is continuous under the same conditions in Theorem 1.8 , which is certainly an interesting issue. 
We would like to remark that the proof of Theorem 1.8 for $\left[b, M_{\alpha}\right]$ is based on Lemma 2.1 and some known regularity results on the Hardy-Littlewood maximal operator and its fractional version (see Lemma 2.4). The main ingredients of proving Theorem 1.8 for $\mathfrak{M}_{b, \alpha}$ are the equivalent characterization of Sobolev spaces (see (2.1)), the characterization of the product of a function in $W^{1, p}\left(\mathbb{R}^{n}\right)$ and a function in $\operatorname{Lip}\left(\mathbb{R}^{n}\right)$ (see Lemma 2.1) and the difference estimates for $\mathfrak{M}_{b, \alpha} f$ (see 3.5 ).

\subsection{The local case}

Let us recall the definitions of the commutators of local fractional maximal operator.

Definition 1.10. Let $\Omega$ be a subdomain in $\mathbb{R}^{n}$ and $0 \leq \alpha<n$. We denote by $M_{\alpha, \Omega}$ the local fractional maximal operator on $\Omega$, i.e., for $f \in L_{\text {loc }}^{1}(\Omega)$,

$$
M_{\alpha, \Omega} f(x)=\sup _{0<r<\operatorname{dist}\left(x, \Omega^{c}\right)} \frac{r^{\alpha}}{|B(x, r)|} \int_{B(x, r)}|f(y)| d y, \quad x \in \Omega,
$$

where $\Omega^{c}=\mathbb{R}^{n} \backslash \Omega$. For a locally integrable function $b$ defined on $\Omega$, we define the commutator of local fractional maximal operator $\left[b, M_{\alpha, \Omega}\right]$ by

$$
\left[b, M_{\alpha, \Omega}\right](f)(x)=b(x) M_{\alpha, \Omega} f(x)-M_{\alpha, \Omega}(b f)(x), \quad x \in \Omega .
$$

The fractional maximal commutator of $M_{\alpha, \Omega}$ with $b$ is defined by

$$
\mathfrak{M}_{b, \alpha, \Omega} f(x)=\sup _{0<r<\operatorname{dist}\left(x, \Omega^{c}\right)} \frac{r^{\alpha}}{|B(x, r)|} \int_{B(x, r)}|b(x)-b(y)||f(y)| d y, \quad x \in \Omega
$$

It is clear that $\left[b, M_{\alpha, \Omega}\right]=\left[b, M_{\alpha}\right]$ and $\mathfrak{M}_{b, \alpha, \Omega}=\mathfrak{M}_{b, \alpha}$ when $\Omega=\mathbb{R}^{n}$. When $\alpha=0$, we denote $\left[b, M_{\alpha, \Omega}\right]=\left[b, M_{\Omega}\right]$ and $\mathfrak{M}_{b, \alpha, \Omega}=\mathfrak{M}_{b, \Omega}$, the operator $M_{\alpha, \Omega}$ reduces to the usual local Hardy-Littlewood maximal operator $M_{\Omega}$.

The following are some basic properties for $\left[b, M_{\alpha, \Omega}\right]$ and $\mathfrak{M}_{b, \alpha, \Omega}$, which are useful for our proofs.

Remark 1.11. Let $1<p<\infty, 0 \leq \alpha<n / p$ and $1 / q=1 / p-\alpha / n$. Assume that $b \in L^{\infty}(\Omega)$. The following facts are valid:

(i) If $f \in L^{p}(\Omega)$, then

$$
\left\|M_{\alpha, \Omega} f\right\|_{q, \Omega} \leq C_{\alpha, n, p}\|f\|_{p, \Omega}
$$

By 1.10 and the sublinearity of $M_{\alpha, \Omega}$, we see that $M_{\alpha, \Omega}: L^{p}(\Omega) \rightarrow L^{q}(\Omega)$ is continuous. 
(ii) The operator $\left[b, M_{\alpha, \Omega}\right]$ is neither positive nor sublinear. Applying Hölder's inequality and 1.10 , one may get

$$
\left\|\left[b, M_{\alpha, \Omega}\right](f)\right\|_{q, \Omega} \leq C_{\alpha, n, p}\|b\|_{\infty, \Omega}\|f\|_{p, \Omega} .
$$

It is not difficult to see that

$$
\left|\left[b, M_{\alpha, \Omega}\right](f)-\left[b, M_{\alpha, \Omega}\right](g)\right| \leq|b| M_{\alpha, \Omega}(f-g)+M_{\alpha, \Omega}(b(f-g)),
$$

which together with 1.10 implies that $\left[b, M_{\alpha, \Omega}\right]: L^{p}(\Omega) \rightarrow L^{q}(\Omega)$ is continuous.

(iii) The operator $\mathfrak{M}_{b, \alpha, \Omega}$ is positive and sublinear. Observe that

$$
\mathfrak{M}_{b, \alpha, \Omega} f(x) \leq|b(x)| M_{\alpha, \Omega} f(x)+M_{\alpha, \Omega}(b f)(x), \quad x \in \Omega .
$$

Inequality 1.12 together with Hölder's inequality and 1.10 implies that

$$
\left\|\mathfrak{M}_{b, \alpha, \Omega} f\right\|_{q, \Omega} \leq C_{\alpha, n, p}\|b\|_{\infty, \Omega}\|f\|_{p, \Omega}
$$

Moreover, we get by the sublinearity and boundedness for $\mathfrak{M}_{b, \alpha, \Omega}$ that the map $\mathfrak{M}_{b, \alpha, \Omega}: L^{p}(\Omega) \rightarrow L^{q}(\Omega)$ is continuous.

The Sobolev regularity for maximal operators in local setting has been studied by many authors. The first work was due to Kinnunen and Lindqvist [17] who proved that the map $M_{\Omega}: W^{1, p}(\Omega) \rightarrow W^{1, p}(\Omega)$ is bounded for all $1<p \leq \infty$ (see also $[13)$. Here $W^{1, p}(\Omega)$ is the first order Sobolev space on $\Omega$, which is defined in the same way as $W^{1, p}\left(\mathbb{R}^{n}\right)$, but with $\mathbb{R}^{n}$ replaced by $\Omega$. For simplicity, we denote

$$
\|f\|_{1, p, \Omega}:=\|f\|_{W^{1, p}(\Omega)}, \quad\|f\|_{p, \Omega}:=\|f\|_{L^{p}(\Omega)} .
$$

Later on, the main result of [17] was extended by many authors (see [14, 15, 25, 28]). Particularly, Liu and Xi [25] established the Sobolev regularity of $\left[b, M_{\alpha, \Omega}\right]$ and $\mathfrak{M}_{b, \alpha, \Omega}$. The main results of 25 can be listed as follows:

Theorem 1.12. 25

(i) Let $1<p_{1}, p_{2}, p_{1} p_{2} /\left(p_{1}+p_{2}\right)<\infty, 1<p_{1}<n, 1 \leq \alpha<n / p_{1}$ and $1 / p=$ $1 / p_{1}+1 / p_{2}-(\alpha-1) / n$. Assume that $|\Omega|<\infty$ and $b \in W^{1, p_{2}}(\Omega)$, then the map $\left[b, M_{\alpha, \Omega}\right]: W^{1, p_{1}}(\Omega) \rightarrow W^{1, p}(\Omega)$ is bounded. Moreover, if $f \in W^{1, p_{1}}(\Omega)$, then

$$
\left\|\left[b, M_{\alpha, \Omega}\right](f)\right\|_{1, p, \Omega} \leq C_{\alpha, n, p_{1}, p_{2}, \Omega}\|b\|_{1, p_{2}, \Omega}\|f\|_{1, p_{1}, \Omega} .
$$

(ii) Let $1<p_{1}, p_{2}, p_{1} p_{2} /\left(p_{1}+p_{2}\right)<\infty, 1<p_{1}<n, 1 \leq \alpha<n / p_{1}$ and $1 / p=1 / p_{1}+$ $1 / p_{2}-\alpha / n$. Assume that $b \in W^{1, p_{2}}(\Omega)$ and $\Omega$ admits a $p_{1}$-Sobolev embedding, then the map $\left[b, M_{\alpha, \Omega}\right]: W^{1, p_{1}}(\Omega) \rightarrow W^{1, p}(\Omega)$ is bounded. Moreover, if $f \in W^{1, p_{1}}(\Omega)$, then

$$
\left\|\left[b, M_{\alpha, \Omega}\right](f)\right\|_{1, p, \Omega} \leq C_{\alpha, n, p_{1}, p_{2}}\|b\|_{1, p_{2}, \Omega}\|f\|_{1, p_{1}, \Omega} .
$$


(iii) Let $1<p_{1}, p_{2}<\infty, n /(n-1)<p_{1} p_{2} /\left(p_{1}+p_{2}\right)<\infty, 1 \leq \alpha<n / p_{1}$ and $1 / p=$ $1 / p_{1}+1 / p_{2}-(\alpha-1) / n$. Assume that $|\Omega|<\infty$ and $b \in W^{1, p_{2}}(\Omega)$, then the map $\left[b, M_{\alpha, \Omega}\right]: L^{p_{1}}(\Omega) \rightarrow W^{1, p}(\Omega)$ is bounded. Moreover, if $f \in L^{p_{1}}(\Omega)$, then

$$
\left\|\left[b, M_{\alpha, \Omega}\right](f)\right\|_{1, p, \Omega} \leq C_{\alpha, n, p_{1}, p_{2}, \Omega}\|b\|_{1, p_{2}, \Omega}\|f\|_{p_{1}, \Omega}
$$

The above conclusions hold for $\mathfrak{M}_{b, \alpha, \Omega}$.

Based on Theorems 1.8 and 1.12 , it is natural to ask the following question:

Question 1.13. What happens when we consider the Sobolev regularity properties for $\left[b, M_{\alpha, \Omega}\right]$ and $\mathfrak{M}_{b, \alpha, \Omega}$ when $b$ belongs to local Lipschitz space?

Question 1.13 is another one of main motivations in this paper. Before establishing the rest results, let us introduce local Lipschitz space.

Definition 1.14 (Local Lipschitz space). The homogeneous local Lipschitz space Lip $(\Omega)$ is defined as

$$
\operatorname{Lip}(\Omega):=\left\{f: \Omega \rightarrow \mathbb{C} \text { continuous }:\|f\|_{\operatorname{Lip}(\Omega)}<\infty\right\}
$$

where

$$
\|f\|_{L i p(\Omega)}:=\sup _{x \in \Omega} \sup _{h \in \Omega \backslash\{0\}} \frac{|f(x+h)-f(x)|}{|h|}<\infty .
$$

The inhomogeneous Lipschitz space $\operatorname{Lip}(\Omega)$ is given by

$$
\operatorname{Lip}(\Omega):=\left\{f: \Omega \rightarrow \mathbb{C} \text { continuous }:\|f\|_{\operatorname{Lip}(\Omega)}<\infty\right\},
$$

where

$$
\|f\|_{\operatorname{Lip}(\Omega)}:=\|f\|_{\infty, \Omega}+\|f\|_{\operatorname{Lip}(\Omega)}<\infty .
$$

Remark 1.15. Let $b \in \operatorname{Lip}(\Omega)$. Then the weak partial derivatives $D_{i} b, i=1, \ldots, n$, exist almost everywhere. Moreover, it holds that

$$
D_{i} b(x)=\lim _{h \rightarrow 0} \frac{b\left(x+h e_{i}\right)-b(x)}{h}
$$

and

$$
\left|D_{i} b(x)\right| \leq\|b\|_{\operatorname{Lip}(\Omega)}
$$

for almost every $x \in \Omega$. By (1.14), we get

$$
|\nabla b(x)| \leq \sqrt{n}\|b\|_{\operatorname{Lip}(\Omega)}
$$

for almost every $x \in \Omega$. 
The rest of main results can be listed as follows:

Theorem 1.16. Let $b \in \operatorname{Lip}(\Omega)$.

(i) Let $1<p<\infty$. Then the $\operatorname{map}\left[b, M_{\Omega}\right]: W^{1, p}(\Omega) \rightarrow W^{1, p}(\Omega)$ is bounded and continuous. If $f \in W^{1, p}(\Omega)$, then

$$
\left|\nabla\left[b, M_{\Omega}\right](f)(x)\right| \leq 4\|b\|_{\infty, \Omega} M_{\Omega}|\nabla f|(x)+3 \sqrt{n}\|b\|_{L i p(\Omega)} M_{\Omega} f(x)
$$

for almost every $x \in \Omega$. Consequently,

$$
\left\|\left[b, M_{\Omega}\right](f)\right\|_{1, p, \Omega} \leq C_{n, p}\|b\|_{\operatorname{Lip}(\Omega)}\|f\|_{1, p, \Omega}
$$

(ii) Let $p \in(1, n), \alpha \in[1, n / p)$ and $q=n p /(n-(\alpha-1) p)$. Assume that $|\Omega|<\infty$, then $\left[b, M_{\alpha, \Omega}\right]: W^{1, p}(\Omega) \rightarrow W^{1, q}(\Omega)$ is bounded and continuous. In particular, if $f \in W^{1, p}(\Omega)$, then

$$
\begin{aligned}
& \left|\nabla\left[b, M_{\alpha, \Omega}\right](f)(x)\right| \\
\leq & 3 \sqrt{n}\|b\|_{L i p(\Omega)} M_{\alpha, \Omega} f(x)+4\|b\|_{\infty, \Omega} M_{\alpha, \Omega}|\nabla f|(x)+2 \alpha\|b\|_{\infty, \Omega} M_{\alpha-1, \Omega} f(x)
\end{aligned}
$$

for almost every $x \in \Omega$. Consequently,

$$
\left\|\left[b, M_{\alpha, \Omega}\right](f)\right\|_{1, q, \Omega} \leq C_{\alpha, n, p,|\Omega|}\|b\|_{\operatorname{Lip}(\Omega)}\|f\|_{1, p, \Omega} .
$$

(iii) Let $p \in(1, n), \alpha \in[1, n / p)$ and $q=p n /(n-\alpha p)$. If $f \in W^{1, p}(\Omega)$ and $\Omega$ admits a $p$ Sobolev embedding, i.e., $\|f\|_{\tilde{p}, \Omega} \leq C_{p}\|f\|_{1, p, \Omega}$ with $\widetilde{p}=n p /(n-p)$, then $\left[b, M_{\alpha, \Omega}\right](f) \in$ $W^{1, q}(\Omega)$. Moreover,

$$
\begin{aligned}
& \left|\nabla\left[b, M_{\alpha, \Omega}\right](f)(x)\right| \\
\leq & 3 \sqrt{n}\|b\|_{L i p(\Omega)} M_{\alpha, \Omega} f(x)+4\|b\|_{\infty, \Omega} M_{\alpha, \Omega}|\nabla f|(x)+2 \alpha\|b\|_{\infty, \Omega} M_{\alpha-1, \Omega} f(x)
\end{aligned}
$$

for almost every $x \in \Omega$. Consequently,

$$
\left\|\left[b, M_{\alpha, \Omega}\right](f)\right\|_{1, q, \Omega} \leq C_{\alpha, n, p}\|b\|_{\operatorname{Lip}(\Omega)}\|f\|_{1, p, \Omega} .
$$

(iv) Let $p \in(n /(n-1), \infty), \alpha \in[1, \min \{(n-1) / p, n-2 n /((n-1) p)\}+1), q=n p /(n-$ $(\alpha-1) p)$ and $|\Omega|<\infty$. If $f \in L^{p}(\Omega)$, then

$$
\begin{aligned}
& \left|\nabla\left[b, M_{\alpha, \Omega}\right](f)(x)\right| \\
\leq & \sqrt{n}\|b\|_{L i p(\Omega)} M_{\alpha, \Omega} f(x)+C_{n}\|b\|_{\infty, \Omega}\left(M_{\alpha-1, \Omega} f(x)+\mathcal{S}_{\alpha-1, \Omega} f(x)\right)
\end{aligned}
$$

for almost every $x \in \Omega$. Moreover,

$$
\left\|\left[b, M_{\alpha, \Omega}\right](f)\right\|_{1, q, \Omega} \leq C_{\alpha, n, p,|\Omega|}\|b\|_{\operatorname{Lip}(\Omega)}\|f\|_{p, \Omega} .
$$


Here $\mathcal{S}_{\alpha, \Omega}$ is the local spherical maximal operator, i.e.,

$$
\mathcal{S}_{\alpha, \Omega} f(x)=\sup _{0<r<\operatorname{dist}\left(x, \Omega^{c}\right)} \frac{r^{\alpha}}{|\partial B(x, r)|} \int_{\partial B(x, r)}|f(y)| d \mathcal{H}^{n-1}(y),
$$

where $d \mathcal{H}^{n-1}$ is the normalized $(n-1)$-dimensional Hausdorff measure.

Theorem 1.17. Let $b \in \operatorname{Lip}(\Omega)$.

(i) Let $1<p<\infty$. Then the map $\mathfrak{M}_{b, \Omega}: W^{1, p}(\Omega) \rightarrow W^{1, p}(\Omega)$ is bounded. If $f \in$ $W^{1, p}(\Omega)$, then

$$
\left|\nabla \mathfrak{M}_{b, \Omega} f(x)\right| \leq 3 \sqrt{n}\|b\|_{L i p(\Omega)} M_{\Omega} f(x)+2 \mathfrak{M}_{b, \Omega}|\nabla f|(x)
$$

for almost every $x \in \Omega$. Consequently,

$$
\left\|\mathfrak{M}_{b, \Omega} f\right\|_{1, p, \Omega} \leq C_{n, p}\|b\|_{\operatorname{Lip}(\Omega)}\|f\|_{1, p, \Omega} .
$$

(ii) Let $p \in(1, n), \alpha \in[1, n / p)$ and $q=n p /(n-(\alpha-1) p)$. Assume that $|\Omega|<\infty$, then $\mathfrak{M}_{b, \alpha, \Omega}: W^{1, p}(\Omega) \rightarrow W^{1, q}(\Omega)$ is bounded. In particular, if $f \in W^{1, p}(\Omega)$, then

(1.26) $\left|\nabla \mathfrak{M}_{b, \alpha, \Omega} f(x)\right| \leq \alpha \mathfrak{M}_{b, \alpha-1, \Omega} f(x)+3 \sqrt{n}\|b\|_{L i p(\Omega)} M_{\alpha, \Omega} f(x)+2 \mathfrak{M}_{b, \alpha, \Omega}|\nabla f|(x)$

for almost every $x \in \Omega$. Consequently,

$$
\left\|\mathfrak{M}_{b, \alpha, \Omega} f\right\|_{1, q, \Omega} \leq C_{\alpha, n, p,|\Omega|}\|b\|_{\operatorname{Lip}(\Omega)}\|f\|_{1, p, \Omega} .
$$

(iii) Let $p \in(1, n), \alpha \in[1, n / p)$ and $q=p n /(n-\alpha p)$. If $f \in W^{1, p}(\Omega)$ and $\Omega$ admits a p-Sobolev embedding, i.e., $\|f\|_{\widetilde{p}, \Omega} \leq C_{p}\|f\|_{1, p, \Omega}$ with $\widetilde{p}=n p /(n-p)$, then $\mathfrak{M}_{b, \alpha, \Omega} f \in$ $W^{1, q}(\Omega)$. Moreover,

$$
\left|\nabla \mathfrak{M}_{b, \alpha, \Omega} f(x)\right| \leq \alpha \mathfrak{M}_{b, \alpha-1, \Omega} f(x)+2 \mathfrak{M}_{b, \alpha, \Omega}|\nabla f|(x)+2 \sqrt{n}\|b\|_{L i p(\Omega)} M_{\alpha, \Omega} f(x)
$$

for almost every $x \in \Omega$. Consequently,

$$
\left\|\mathfrak{M}_{b, \alpha, \Omega} f\right\|_{1, q, \Omega} \leq C_{\alpha, n, p}\|b\|_{\operatorname{Lip}(\Omega)}\|f\|_{1, p, \Omega} .
$$

(iv) Let $p \in(n /(n-1), \infty), \alpha \in[1, \min \{(n-1) / p, n-2 n /((n-1) p)\}+1)$ and $|\Omega|<\infty$. If $f \in L^{p}(\Omega)$, then

$$
\begin{aligned}
& \left|\nabla \mathfrak{M}_{b, \alpha, \Omega} f(x)\right| \\
\leq & (n-\alpha) \mathfrak{M}_{b, \alpha-1, \Omega} f(x)+\sqrt{n}\|b\|_{L i p(\Omega)} M_{\alpha, \Omega} f(x)+2 n\|b\|_{\infty, \Omega} \mathcal{S}_{\alpha-1, \Omega} f(x)
\end{aligned}
$$

for almost every $x \in \Omega$. Moreover,

$$
\left\|\mathfrak{M}_{b, \alpha, \Omega} f\right\|_{1, q, \Omega} \leq C_{\alpha, n, p,|\Omega|}\|b\|_{\operatorname{Lip}(\Omega)}\|f\|_{p, \Omega},
$$

where $q=n p /(n-(\alpha-1) p)$. 
The proof of Theorem 1.16 is based on Lemmas 2.5 and 2.6 and a characterization of the product of a function in $W^{1, p}(\Omega)$ and a function in $\operatorname{Lip}(\Omega)$ (see Lemma 2.3). The proof of Theorem 1.17 is motivated by the idea in $14,15,25$. However, some new techniques and more refined analyses are needed in the proof of Theorem 1.17 .

Finally, we shall show that the above commutators preserve the zero boundary values in Sobolev's sense. Recall that the Sobolev space $W_{0}^{1, p}(\Omega)$ with zero boundary values with $1 \leq p<\infty$, is defined as the completion of $\mathcal{C}_{0}^{\infty}(\Omega)$ with respect to the Sobolev norm. In 1998, Kinnunen and Lindqvist 17 first proved that $M_{\Omega}$ is bounded on $W_{0}^{1, p}(\Omega)$ with $1<p<\infty$. Later on, Heikkinen, Kinnunen, Korvenpää and Tuominen 15 proved that $M_{\alpha, \Omega}$ is bounded from $L^{p}(\Omega)$ to $W_{0}^{1, q}(\Omega)$ for $p>n /(n-1), 1 \leq \alpha<n / p$ and $q=n p /(n-(\alpha-1) p)$ by assuming that $|\Omega|<\infty$. Recently, by assuming that $|\Omega|<\infty$, Hart, Liu and Xue 14 established the boundedness for $M_{\alpha, \Omega}: W^{1, p}(\Omega) \rightarrow W_{0}^{1, q}(\Omega)$ for $1<p, q<\infty, q=n p /(n-(\alpha-1) p), 1 \leq \alpha<n / p+1$.

As direct applications of Theorems 1.16 and 1.17 , the following conclusions are valid.

Corollary 1.18. Let $b \in \operatorname{Lip}(\Omega)$.

(i) Let $1<p<\infty$. If $f \in W_{0}^{1, p}(\Omega)$, then $\left[b, M_{\Omega}\right](f) \in W_{0}^{1, p}(\Omega)$.

(ii) Let $p \in(1, n), \alpha \in[1, n / p)$ and $q=n p /(n-(\alpha-1) p)$. Assume that $|\Omega|<\infty$, then $\left[b, M_{\alpha, \Omega}\right]: W^{1, p}(\Omega) \rightarrow W_{0}^{1, q}(\Omega)$ is bounded.

(iii) Let $p \in(1, n), \alpha \in[1, n / p)$ and $q=p n /(n-\alpha p)$. If $f \in W^{1, p}(\Omega)$ and $\Omega$ admits a p-Sobolev embedding, then the map $\left[b, M_{\alpha, \Omega}\right]: W^{1, p}(\Omega) \rightarrow W_{0}^{1, q}(\Omega)$ is bounded.

(iv) Let $p \in(n /(n-1), \infty), \alpha \in[1, \min \{(n-1) / p, n-2 n /((n-1) p)\}+1), q=n p /(n-$ $(\alpha-1) p)$ and $|\Omega|<\infty$, then the $\operatorname{map}\left[b, M_{\alpha, \Omega}\right]: L^{p}(\Omega) \rightarrow W_{0}^{1, q}(\Omega)$ is bounded.

The same conclusions hold for the operator $\mathfrak{M}_{b, \alpha, \Omega}$.

\subsection{Outline of this paper}

This paper will be organized as follows. Section 2 contains some auxiliary notations and lemmas, which paly key roles in the proofs of Theorems 1.8, 1.16 and 1.17. In Section 3 we shall prove Theorem 1.8. The proofs for Theorems 1.16 and 1.17 will be given in Sections 4 and 5 , respectively. Finally, we shall prove Corollary 1.18 in Section 6 .

\section{Preliminary notations and lemmas}

This section is devoted to presenting some notations and lemmas. 


\subsection{Preliminary notations}

Throughout the paper, the letter $C_{\alpha, \beta}$ denote the positive constants that depend on the parameters $\alpha, \beta$. Let $f \in L^{p}\left(\mathbb{R}^{n}\right)$ with $p \geq 1$. For all $h \in \mathbb{R},|h|>0, y \in \mathbb{R}^{n}$ and $i=1, \ldots, n$, we define the function $f_{h, i}$ by setting

$$
f_{h, i}(x)=\frac{f\left(x+h e_{i}\right)-f(x)}{|h|}, \quad x \in \mathbb{R}^{n} .
$$

It is well known that for $p \geq 1, f_{h, i} \rightarrow D_{i} f$ in $L^{p}\left(\mathbb{R}^{n}\right)$ when $h \rightarrow 0$ if $f \in W^{1, p}\left(\mathbb{R}^{n}\right)$. For $h \in \mathbb{R}^{n}$ and any arbitrary functions $f$ defined on $\mathbb{R}^{n}$, we define the first order difference of $f$ by

$$
\Delta_{h} f(x):=f(x+h)-f(x), \quad x \in \mathbb{R}^{n} .
$$

For $y \in \mathbb{R}^{n}$, we define the function $f_{y}$ by $f_{y}(x)=f(x+y)$. Set

$$
G(f ; p)=\limsup _{h \rightarrow 0} \frac{\left\|\Delta_{h} f\right\|_{p}}{|h|} .
$$

According to [11, Section 7.11], we have

$$
u \in W^{1, q}\left(\mathbb{R}^{n}\right), \quad 1<q<\infty \quad \Longleftrightarrow u \in L^{q}\left(\mathbb{R}^{n}\right) \text { and } G(u ; q)<\infty .
$$

\subsection{Some lemmas}

The following result presents a characterization of the product of a function in $W^{1, p}\left(\mathbb{R}^{n}\right)$ and a function in $\operatorname{Lip}\left(\mathbb{R}^{n}\right)$.

Lemma 2.1. Let $1<p<\infty$. If $f \in W^{1, p}\left(\mathbb{R}^{n}\right)$ and $b \in \operatorname{Lip}\left(\mathbb{R}^{n}\right)$, then bf $\in W^{1, p}\left(\mathbb{R}^{n}\right)$. Moreover,

$$
D_{i}(b f)=b D_{i} f+f D_{i} b, \quad i=1, \ldots, n
$$

almost everywhere in $\mathbb{R}^{n}$. Consequently,

$$
\nabla(b f)=b \nabla f+f \nabla b
$$

almost everywhere in $\mathbb{R}^{n}$. In particular, it holds that

$$
\|b f\|_{1, p} \leq \sqrt{n}\|b\|_{\operatorname{Lip}\left(\mathbb{R}^{n}\right)}\|f\|_{1, p} .
$$

Proof. The proof is similar to that of Lemma 2.1 in $[26$. At first, we claim that bf $\in$ $W^{1, p}\left(\mathbb{R}^{n}\right)$. It is easy to see that

$$
\|b f\|_{p} \leq\|b\|_{\infty}\|f\|_{p}
$$


One can easily check that

$$
\Delta_{h}(b f)=(b f)_{h}-b f=b_{h} \Delta_{h} f+f \Delta_{h} b
$$

for any $h \in \mathbb{R}^{n}$. It follows from $(2.6)$ that

$$
G(b f ; p)=\limsup _{h \rightarrow 0} \frac{\left\|\Delta_{h}(b f)\right\|_{p}}{|h|} \leq\|b\|_{\infty} G(f ; p)+\|b\|_{L i p\left(\mathbb{R}^{n}\right)}\|f\|_{p}<\infty .
$$

This together with (2.1) and (2.5) yields that $b f \in W^{1, p}\left(\mathbb{R}^{n}\right)$.

Next we shall prove (2.2). Fix $i \in\{1, \ldots, n\}$. Noting that $f_{h, i} \rightarrow D_{i} f$ and $(b f)_{h, i} \rightarrow$ $D_{i}(b f)$ in $L^{p}\left(\mathbb{R}^{n}\right)$ when $h \rightarrow 0$. This together with (1.1) yields that there exist a sequence of numbers $\left\{h_{k}\right\}$ satisfying $\lim _{k \rightarrow \infty} h_{k}=0$ and a measurable set $E$ satisfying $\left|\mathbb{R}^{n} \backslash E\right|=0$ such that $f_{h_{k}, i}(x) \rightarrow D_{i} f(x),(b f)_{h_{k}, i}(x) \rightarrow D_{i}(b f)(x)$ and $b_{h_{k}, i}(x) \rightarrow D_{i} b(x)$ as $k \rightarrow \infty$ for all $x \in E$. It is easy to see that $b\left(h_{k} e_{i}+x\right) \rightarrow b(x)$ as $k \rightarrow \infty$ for all $x \in E$ since $\left|b\left(h_{k} e_{i}+x\right)-b(x)\right| \leq\|b\|_{L i p\left(\mathbb{R}^{n}\right)}\left|h_{k}\right|$.

Therefore, we get from (2.6) that

$$
\begin{aligned}
D_{i}(b f)(x) & =\lim _{k \rightarrow \infty}(b f)_{h_{k}, i}(x) \\
& =\lim _{k \rightarrow \infty}\left(b\left(h_{k} e_{i}+x\right) f_{h_{k}, i}(x)+b_{h_{k}, i}(x) f(x)\right)=b(x) D_{i} f(x)+f(x) D_{i} b(x)
\end{aligned}
$$

for any $x \in E$. This proves (2.2). Equality (2.3) follows easily from (2.2). By (2.3), (2.5), (1.3) and Minkowski's inequality, we have

$$
\|b f\|_{1, p}=\|b f\|_{p}+\|\nabla(b f)\|_{p} \leq\|b\|_{\infty}\|f\|_{p}+\|b \nabla f\|_{p}+\|f \nabla b\|_{p} \leq \sqrt{n}\|b\|_{\operatorname{Lip}\left(\mathbb{R}^{n}\right)}\|f\|_{1, p},
$$

which gives (2.4) and completes the proof.

Proposition 2.2. [15, 17] Let $1 \leq p \leq \infty$. If $f_{k} \rightarrow f, g_{k} \rightarrow g$ weakly in $L^{p}(\Omega)$ and $f_{k} \leq g_{k}(k=1,2, \ldots)$ almost everywhere in $\Omega$, then $f \leq g$ almost everywhere in $\Omega$.

Applying Proposition 2.2, we can get a local version of Lemma 2.1.

Lemma 2.3. Let $1<p<\infty$. If $b \in \operatorname{Lip}(\Omega)$ and $f \in W^{1, p}(\Omega)$, then $b f \in W^{1, p}(\Omega)$. Moreover,

$$
\nabla(b f)=b \nabla f+f \nabla b
$$

almost everywhere in $\Omega$. In particular, it holds that

$$
\|b f\|_{1, p, \Omega} \leq \sqrt{n}\|b\|_{\operatorname{Lip}(\Omega)}\|f\|_{1, p, \Omega} .
$$


Proof. Since $f \in W^{1, p}(\Omega)$, there exists a sequence $\left\{\varphi_{j}\right\}_{j=1}^{\infty}$ of functions in $W^{1, p}(\Omega) \cap \mathcal{C}^{\infty}(\Omega)$ such that $\varphi_{j} \rightarrow f$ in $W^{1, p}(\Omega)$ as $j \rightarrow \infty$. Fix $j \in \mathbb{N}$. Note that $b$ is differentiable almost everywhere in $\Omega$. By Leibniz rule,

$$
D_{i}\left(\varphi_{j} b\right)(x)=D_{i} \varphi_{j}(x) b(x)+D_{i} b(x) \varphi_{j}(x)
$$

for all $i=1, \ldots, n$ and almost every $x \in \Omega$. Thus we have

$$
\nabla\left(\varphi_{j} b\right)(x)=b(x) \nabla \varphi_{j}(x)+\varphi_{j}(x) \nabla b(x)
$$

for almost every $x \in \Omega$. By 2.9), 1.15) and Minkowski's inequality, one finds that

$$
\begin{aligned}
\left\|\varphi_{j} b\right\|_{1, p, \Omega} & =\left\|\varphi_{j} b\right\|_{p, \Omega}+\left\|\nabla\left(\varphi_{j} b\right)\right\|_{p, \Omega} \\
& \leq\|b\|_{\infty, \Omega}\left\|\varphi_{j}\right\|_{p, \Omega}+\left\|\nabla \varphi_{j} b\right\|_{p, \Omega}+\left\|\nabla b \varphi_{j}\right\|_{p, \Omega} \leq \sqrt{n}\|b\|_{\operatorname{Lip}(\Omega)}\left\|\varphi_{j}\right\|_{1, p, \Omega}
\end{aligned}
$$

and

$$
\begin{aligned}
\left\|\nabla\left(\varphi_{j} b\right)-(b(\nabla f)+f(\nabla b))\right\|_{p, \Omega} & \leq\left\|b \nabla \varphi_{j}-b \nabla f\right\|_{p, \Omega}+\left\|\varphi_{j} \nabla b-f \nabla b\right\|_{p, \Omega} \\
& \leq \sqrt{n}\|b\|_{\operatorname{Lip}(\Omega)}\left\|\varphi_{j}-f\right\|_{1, p, \Omega} .
\end{aligned}
$$

It follows from 2.10 and $(2.11)$ that $\left\{\varphi_{j} b\right\}_{j=1}^{\infty}$ is a bounded sequence in $W^{1, p}(\Omega)$ and

$$
\nabla\left(\varphi_{j} b\right) \rightarrow b \nabla f+f \nabla b \quad \text { in } L^{p}(\Omega) \text { as } j \rightarrow \infty .
$$

Since $\varphi_{j} b \rightarrow f b$ in $L^{p}(\Omega)$ as $j \rightarrow \infty$, then by Riesz theorem, there exists a subsequence $\left\{\varphi_{j_{k}} b\right\}_{k=1}^{\infty}$ such that

$$
\varphi_{j_{k}}(x) b(x) \rightarrow f(x) b(x) \quad \text { as } k \rightarrow \infty
$$

for almost every $x \in \Omega$. Consequently, there exists a measurable set $E$ such that $|\Omega \backslash E|=0$ and

$$
\varphi_{j_{k}}(x) b(x) \rightarrow f(x) b(x) \quad \text { as } k \rightarrow \infty
$$

for every $x \in E$. From the above we can conclude that the Sobolev derivative $\nabla(f b)$ exists almost everywhere in $E$ and that

$$
\nabla\left(\varphi_{j} b\right) \rightarrow \nabla(f b) \quad \text { weakly in } L^{p}(E) \text { as } j \rightarrow \infty .
$$

Furthermore, the weak gradient $\nabla(f b)$ exists almost everywhere in $\Omega$ and

$$
\nabla\left(\varphi_{j} b\right) \rightarrow \nabla(f b) \quad \text { weakly in } L^{p}(\Omega) \text { as } j \rightarrow \infty \text {. }
$$

Applying Proposition 2.2 and (2.12), 2.13), we can get (2.7). By (2.7), (1.15) and Minkowski's inequality, one can get

$$
\begin{aligned}
\|b f\|_{1, p, \Omega} & =\|b f\|_{p, \Omega}+\|\nabla(b f)\|_{p, \Omega} \\
& \leq\|b\|_{\infty, \Omega}\|f\|_{p, \Omega}+\left(\|b\|_{\infty, \Omega}\|\nabla f\|_{p, \Omega}+\sqrt{n}\|b\|_{\operatorname{Lip}(\Omega)}\|f\|_{p, \Omega}\right) \\
& \leq \sqrt{n}\|b\|_{\operatorname{Lip}(\Omega)}\|f\|_{1, p, \Omega},
\end{aligned}
$$

which gives 2.8. 
In order to prove Theorem 1.8 for the commutator $\left[b, M_{\alpha}\right]$, we need the following known results.

Lemma 2.4. [16, 19,22, 27] Let $1<p<\infty, 0 \leq \alpha<n / p$ and $q=n p /(n-\alpha p)$. Then the map $M_{\alpha}: W^{1, p}\left(\mathbb{R}^{n}\right) \rightarrow W^{1, q}\left(\mathbb{R}^{n}\right)$ is bounded and continuous. Moreover, if $f \in W^{1, p}\left(\mathbb{R}^{n}\right)$, then

$$
\left|D_{i} M_{\alpha} f(x)\right| \leq M_{\alpha} D_{i} f(x), \quad i=1,2, \ldots, n
$$

for almost every $x \in \mathbb{R}^{n}$. Consequently,

$$
\left\|M_{\alpha} f\right\|_{1, q} \leq C_{p, q, n}\|f\|_{1, p} .
$$

The boundedness part and pointwise estimates in Lemma 2.4 for $\alpha=0$ (resp., $0<\alpha<$ $n$ ) follows from [16, Theorem 1.4] (resp., [19, Theorem 2.1] and [19, Remark 2.2]). The continuity part in Lemma 2.4 for $\alpha=0$ (resp., $0<\alpha<n$ ) follows from [27, Theorem 4.1] (resp., 22, Remark 1]).

The following known results are the main ingredients of proving Theorem 1.16 .

Lemma 2.5. (i) [17] Let $1<p<\infty$. Then the map $M_{\Omega}: W^{1, p}(\Omega) \rightarrow W^{1, p}(\Omega)$ is bounded. Moreover, if $f \in W^{1, p}(\Omega)$, then

$$
\left|\nabla M_{\Omega} f(x)\right| \leq 2 M_{\Omega}|\nabla f|(x)
$$

for almost every $x \in \Omega$.

(ii) 28 The map $M_{\Omega}: W^{1, p}(\Omega) \rightarrow W^{1, p}(\Omega)$ is continuous.

Lemma 2.6. (i) $\quad 15$ Let $p \in(1, n), \alpha \in[1, n / p)$ and $q=n p /(n-(\alpha-1) p)$. If $f \in W^{1, p}(\Omega)$ and $|\Omega|<\infty$, then

$$
\left|\nabla M_{\alpha, \Omega} f(x)\right| \leq 2 M_{\alpha, \Omega}|\nabla f|(x)+\alpha M_{\alpha-1, \Omega} f(x)
$$

for almost every $x \in \Omega$. Consequently,

$$
\left\|M_{\alpha, \Omega} f\right\|_{1, q, \Omega} \leq C_{\alpha, n, p, \Omega}\|f\|_{1, p, \Omega} .
$$

(ii) [14 Let $p \in(1, n), \alpha \in[1, n / p)$ and $q=n p /(n-(\alpha-1) p)$. Assume that $|\Omega|<\infty$, then the map $M_{\alpha, \Omega}: W^{1, p}(\Omega) \rightarrow W^{1, q}(\Omega)$ is continuous.

(iii) [14] Let $p \in(1, n), \alpha \in[1, n / p)$ and $q=p n /(n-\alpha p)$. If $f \in W^{1, p}(\Omega)$ and $\Omega$ admits a p-Sobolev embedding, i.e., $\|f\|_{\widetilde{p}, \Omega} \leq C_{p}\|f\|_{1, p, \Omega}$ with $\widetilde{p}=n p /(n-p)$, then $M_{\alpha, \Omega} f \in W^{1, q}(\Omega)$. Moreover,

$$
\left|\nabla M_{\alpha, \Omega} f(x)\right| \leq 2 M_{\alpha, \Omega}|\nabla f|(x)+\alpha M_{\alpha-1, \Omega} f(x)
$$

for almost every $x \in \Omega$. Consequently,

$$
\left\|M_{\alpha, \Omega} f\right\|_{1, q, \Omega} \leq C_{\alpha, n, p}\|f\|_{1, p, \Omega} .
$$


(iv) 15 Let $p \in(n /(n-1), \infty)$ and $\alpha \in[1, \min \{(n-1) / p, n-2 n /((n-1) p)\}+1)$. If $f \in L^{p}(\Omega)$, then

$$
\left|\nabla M_{\alpha, \Omega} f(x)\right| \leq C_{n}\left(M_{\alpha-1, \Omega} f(x)+\mathcal{S}_{\alpha-1, \Omega} f(x)\right)
$$

for almost every $x \in \Omega$. If in addition $|\Omega|<\infty$, then

$$
\left\|M_{\alpha, \Omega} f\right\|_{1, q, \Omega} \leq C_{\alpha, n, p, \Omega}\|f\|_{1, p, \Omega},
$$

where $q=n p /(n-(\alpha-1) p)$.

In order to prove Theorems 1.16 and 1.17 , the following result is also needed.

Lemma 2.7. 15] Let $n \geq 2, p>n /(n-1), 0 \leq \alpha<\min \{(n-1) / p, n-2 n /((n-1) p)\}$. Then $\left\|\mathcal{S}_{\alpha, \Omega} f\right\|_{q, \Omega} \leq C_{\alpha, n, p}\|f\|_{p, \Omega}$.

\section{Proof of Theorem 1.8}

Throughout this section, we fix $1<p<\infty, 0 \leq \alpha<n / p, 1 / q=1 / p-\alpha / n, f \in W^{1, p}\left(\mathbb{R}^{n}\right)$ and $b \in \operatorname{Lip}\left(\mathbb{R}^{n}\right)$.

\subsection{Proof of Theorem 1.8 for $\left[b, M_{\alpha}\right]$}

The proof of Theorem 1.8 for $\left[b, M_{\alpha}\right]$ will be divided into two steps:

Step 1: Proofs of $(1.8)$ and $(1.9)$. Invoking Lemma 2.1 we note that $b f \in W^{1, p}\left(\mathbb{R}^{n}\right)$ and

$$
D_{i}(b f)=b D_{i} f+f D_{i} b, \quad i=1, \ldots, n
$$

almost everywhere in $\mathbb{R}^{n}$. By Lemma 2.4, we have that $M_{\alpha} f \in W^{1, q}\left(\mathbb{R}^{n}\right)$. This together with Lemma 2.1, (3.1) and Lemma 2.4 implies that

$$
\begin{aligned}
& \left|D_{i}\left(\left[b, M_{\alpha}\right](f)\right)(x)\right| \\
\leq & \left|D_{i}\left(b M_{\alpha} f\right)(x)\right|+\left|D_{i} M_{\alpha}(b f)(x)\right| \\
\leq & \left|D_{i} b(x)\right| M_{\alpha} f(x)+|b(x)|\left|D_{i} M_{\alpha} f(x)\right|+M_{\alpha}\left(D_{i}(b f)\right)(x) \\
\leq & \left|D_{i} b(x)\right| M_{\alpha} f(x)+|b(x)|\left|M_{\alpha} D_{i} f(x)\right|+M_{\alpha}\left(D_{i} b f\right)(x)+M_{\alpha}\left(D_{i} f b\right)(x)
\end{aligned}
$$

for any $i=1, \ldots, n$ and almost every $x \in \mathbb{R}^{n}$. By (3.2) and the arguments similar to those used to derive (2.4) in [16], we have

$$
\left|\nabla\left(\left[b, M_{\alpha}\right](f)\right)(x)\right| \leq|\nabla b(x)| M_{\alpha} f(x)+|b(x)| M_{\alpha}|\nabla f|(x)+M_{\alpha}(|\nabla b| f)(x)+M_{\alpha}(|\nabla f| b)(x)
$$

for almost every $x \in \mathbb{R}^{n}$. This together with 1.3 leads to

$$
\left|\nabla\left(\left[b, M_{\alpha}\right](f)\right)(x)\right| \leq 2 \sqrt{n}\|b\|_{\operatorname{Lip}\left(\mathbb{R}^{n}\right)} M_{\alpha} f(x)+2\|b\|_{\infty} M_{\alpha}|\nabla f|(x)
$$


for almost every $x \in \mathbb{R}^{n}$. This proves (1.8). By (1.8), 1.4, (1.5) and Minkowski's inequality, we have

$$
\begin{aligned}
\left\|\left[b, M_{\alpha}\right](f)\right\|_{1, q} & =\left\|\left[b, M_{\alpha}\right](f)\right\|_{q}+\left\|\nabla\left[b, M_{\alpha}\right](f)\right\|_{q} \\
& \leq 2 A_{\alpha, n, p}\|b\|_{\infty}\|f\|_{p}+2 \sqrt{n}\|b\|_{L i p\left(\mathbb{R}^{n}\right)}\left\|M_{\alpha} f\right\|_{q}+2\|b\|_{L^{\infty}\left(\mathbb{R}^{n}\right)}\left\|M_{\alpha} \mid \nabla f\right\|_{q} \\
& \leq 2 A_{\alpha, n, p} \sqrt{n}\|b\|_{\operatorname{Lip}\left(\mathbb{R}^{n}\right)}\|f\|_{1, p} .
\end{aligned}
$$

This proves (1.9).

Step 2: Proof of the continuity part. Let $f_{j} \rightarrow f$ in $W^{1, p}\left(\mathbb{R}^{n}\right)$ as $j \rightarrow \infty$. By Lemma 2.1 we see that $b f_{j} \rightarrow b f$ in $W^{1, p}\left(\mathbb{R}^{n}\right)$ as $j \rightarrow \infty$. This together with the continuity part of Lemma 2.4 implies that $M_{\alpha}\left(b f_{j}\right) \rightarrow M_{\alpha}(b f)$ in $W^{1, q}\left(\mathbb{R}^{n}\right)$ as $j \rightarrow \infty$. Moreover, $M_{\alpha} f_{j} \rightarrow$ $M_{\alpha} f$ in $W^{1, q}\left(\mathbb{R}^{n}\right)$ as $j \rightarrow \infty$. By Lemma 2.1 again, we have that $b M_{\alpha} f_{j} \rightarrow b M_{\alpha} f$ in $W^{1, q}\left(\mathbb{R}^{n}\right)$ as $j \rightarrow \infty$. Thus, $\left[b, M_{\alpha}\right]\left(f_{j}\right) \rightarrow\left[b, M_{\alpha}\right](f)$ in $W^{1, q}\left(\mathbb{R}^{n}\right)$ as $j \rightarrow \infty$.

\subsection{Proof of Theorem 1.8 for $\mathfrak{M}_{b, \alpha}$}

We divide the proof of Theorem 1.8 for $\mathfrak{M}_{b, \alpha}$ into three steps:

Step 1: Proof of $\mathfrak{M}_{b, \alpha} f \in W^{1, q}\left(\mathbb{R}^{n}\right)$. Fix $x, h \in \mathbb{R}^{n}$, we can write

$$
\left(\mathfrak{M}_{b, \alpha} f\right)_{h}(x)=\sup _{r>0} \frac{r^{\alpha}}{|B(x, r)|} \int_{B(x, r)}\left|b_{h}(x)-b_{h}(y)\right|\left|f_{h}(y)\right| d y,
$$

which leads to

$$
\begin{aligned}
& \left|\left(\mathfrak{M}_{b, \alpha} f\right)_{h}(x)-\mathfrak{M}_{b, \alpha} f(x)\right| \\
\leq & \sup _{r>0} \frac{r^{\alpha}}{|B(x, r)|} \int_{B(x, r)}\left|\left(b_{h}(x)-b_{h}(y)\right) f_{h}(y)-(b(x)-b(y)) f(y)\right| d y .
\end{aligned}
$$

Observe that

$$
\begin{aligned}
& \left(b_{h}(x)-b_{h}(y)\right) f_{h}(y)-(b(x)-b(y)) f(y) \\
= & \left(b_{h}(x)-b_{h}(y)\right)\left(f_{h}(y)-f(y)\right)+\left(b_{h}(x)-b(x)\right) f(y)-\left(b_{h}(y)-b(y)\right) f(y),
\end{aligned}
$$

which together with 3.3 and 1.6 implies that

$$
\begin{aligned}
\left|\Delta_{h}\left(\mathfrak{M}_{b, \alpha} f\right)(x)\right| \leq & \mathfrak{M}_{b_{h}, \alpha}\left(f_{h}-f\right)(x)+\left|b_{h}(x)-b(x)\right| M_{\alpha} f(x)+M_{\alpha}\left(\left(b_{h}-b\right) f\right)(x) \\
\leq & |b(x+h)| M_{\alpha}\left(\Delta_{h} f\right)(x)+\left|\Delta_{h} b(x)\right| M_{\alpha} f(x)+M_{\alpha}\left(\Delta_{h} b \Delta_{h} f\right)(x) \\
& +M_{\alpha}\left(\Delta_{h} b f\right)(x)+M_{\alpha}\left(\Delta_{h} f b\right)(x)
\end{aligned}
$$

for any $h, x \in \mathbb{R}^{n}$. By (3.4), (1.4) and Minkowski's inequality, one has

$$
\begin{aligned}
\left\|\Delta_{h}\left(\mathfrak{M}_{b, \alpha} f\right)\right\|_{q} \leq & \left\|b(\cdot+h) M_{\alpha}\left(\Delta_{h} f\right)\right\|_{q}+\left\|\Delta_{h} b M_{\alpha} f\right\|_{q}+\left\|M_{\alpha}\left(\Delta_{h} b \Delta_{h} f\right)\right\|_{q} \\
& +\left\|M_{\alpha}\left(\Delta_{h} b f\right)\right\|_{q}+\left\|M_{\alpha}\left(\Delta_{h} f b\right)\right\|_{q} \\
\leq & \|b\|_{\infty}\left\|M_{\alpha}\left(\Delta_{h} f\right)\right\|_{q}+\|b\|_{\operatorname{Lip}\left(\mathbb{R}^{n}\right)}\left\|M_{\alpha} f\right\|_{q}|h| \\
& +A_{\alpha, p, n}\left(\left\|\Delta_{h} b \Delta_{h} f\right\|_{p}+\left\|\Delta_{h} b f\right\|_{p}+\left\|\Delta_{h} f b\right\|_{p}\right) \\
\leq & 4 A_{\alpha, p, n}\left(\|b\|_{\operatorname{Lip}\left(\mathbb{R}^{n}\right)}\left\|\Delta_{h} f\right\|_{p}+\|b\|_{L i p\left(\mathbb{R}^{n}\right)}\|f\|_{p}|h|\right),
\end{aligned}
$$


which combining with $G(f ; p)<\infty$ leads to

$$
G\left(\mathfrak{M}_{b, \alpha} f ; q\right)=\limsup _{h \rightarrow 0} \frac{\left\|\Delta_{h}\left(\mathfrak{M}_{b, \alpha} f\right)\right\|_{q}}{|h|} \leq 4 A_{p, q}\|b\|_{\operatorname{Lip}\left(\mathbb{R}^{n}\right)}\left(G(f ; p)+\|f\|_{p}\right)<\infty .
$$

On the other hand, by (1.7) we known that $\mathfrak{M}_{b, \alpha} f \in L^{q}\left(\mathbb{R}^{n}\right)$. This together with (3.6) and (2.1) implies that $\mathfrak{M}_{b, \alpha} f \in W^{1, q}\left(\mathbb{R}^{n}\right)$.

Step 2: Estimate for $\nabla \mathfrak{M}_{b, \alpha}(f)$. We want to show that

$$
\left|\nabla\left(\mathfrak{M}_{b, \alpha} f\right)(x)\right| \leq 2 \sqrt{n}\|b\|_{L i p\left(\mathbb{R}^{n}\right)} M_{\alpha} f(x)+2\|b\|_{\infty} M_{\alpha}|\nabla f|(x)
$$

for almost every $x \in \mathbb{R}^{n}$. To prove $(3.7)$, it suffices to show that

$$
\left|D_{i}\left(\mathfrak{M}_{b, \alpha} f\right)(x)\right| \leq\left|D_{i} b(x)\right| M_{\alpha} f(x)+|b(x)| M_{\alpha} D_{i} f(x)+M_{\alpha}\left(D_{i} b f\right)(x)+M_{\alpha}\left(D_{i} f b\right)(x)
$$

for any $i=1, \ldots, n$ and almost every $x \in \mathbb{R}^{n}$. Once (3.8) was proved, by (3.8) and using the arguments similar to those used in deriving (2.4) in [16, we have

$$
\left|\nabla\left(\mathfrak{M}_{b, \alpha} f\right)(x)\right| \leq|b(x)| M_{\alpha}|\nabla f|(x)+|\nabla b(x)| M_{\alpha} f(x)+M_{\alpha}(|\nabla b| f)(x)+M_{\alpha}(|\nabla f| b)(x)
$$

for almost every $x \in \mathbb{R}^{n}$, which together with 1.3 leads to 3.7 .

Now we prove $(3.8)$. Fix $i \in\{1, \ldots, n\}$. Since $f \in W^{1, p}\left(\mathbb{R}^{n}\right)$ and $\mathfrak{M}_{b, \alpha} f \in W^{1, q}\left(\mathbb{R}^{n}\right)$, then $f_{h, i} \rightarrow D_{i} f$ in $L^{p}\left(\mathbb{R}^{n}\right)$ when $h \rightarrow 0$ and $\left(\left[b, M_{\alpha}\right](f)\right)_{h, i} \rightarrow D_{i}\left(\left[b, M_{\alpha}\right](f)\right)$ in $L^{q}\left(\mathbb{R}^{n}\right)$ when $h \rightarrow 0$. It is clear that $f_{h, i} b \rightarrow D_{i} f b$ in $L^{p}\left(\mathbb{R}^{n}\right)$ when $h \rightarrow 0$. By Remark 1.7(i), we have that $M_{\alpha}\left(f_{h, i}\right) \rightarrow M_{\alpha}\left(D_{i} f\right)$ and $M_{\alpha}\left(f_{h, i} b\right) \rightarrow M_{\alpha}\left(D_{i} f b\right)$ in $L^{q}\left(\mathbb{R}^{n}\right)$ as $h \rightarrow 0$. By (1.2) and $(1.3)$ we have that $\left|D_{i} b(x)\right| \leq\|b\|_{\operatorname{Lip}\left(\mathbb{R}^{n}\right)}$ and $b_{h, i}(x) \rightarrow D_{i} b(x)$ as $h \rightarrow 0$ for almost every $x \in \mathbb{R}^{n}$. It follows that $b_{h, i}(x) f(x) \rightarrow D_{i} b(x) f(x)$ as $h \rightarrow 0$ for almost every $x \in \mathbb{R}^{n}$. By Fatou lemma, we have that $b_{h, i} f \rightarrow D_{i} b f$ in $L^{p}\left(\mathbb{R}^{n}\right)$ as $h \rightarrow 0$. This together with Remark 1.7(i) implies that $M_{\alpha}\left(b_{h, i} f\right) \rightarrow M_{\alpha}\left(D_{i} b f\right)$ in $L^{q}\left(\mathbb{R}^{n}\right)$ as $h \rightarrow 0$. Therefore, there exist a sequence $\left\{h_{k}\right\}$ satisfying $h_{k}>0$ and $\lim _{k \rightarrow 0} h_{k}=0$ and a measurable set $E$ with $\left|\mathbb{R}^{n} \backslash E\right|=0$ such that for all $x \in E$,

(i) $\left(\mathfrak{M}_{b, \alpha} f\right)_{h_{k}, i}(x) \rightarrow D_{i}\left(\mathfrak{M}_{b, \alpha} f\right)(x)$ as $k \rightarrow \infty$ and $b_{h_{k}, i}(x) \rightarrow D_{i} b(x)$ as $k \rightarrow \infty$;

(ii) $M_{\alpha}\left(f_{h_{k}, i}\right)(x) \rightarrow M_{\alpha}\left(D_{i} f\right)(x)$ as $k \rightarrow \infty, M_{\alpha}\left(f_{h_{k}, i} b\right)(x) \rightarrow M_{\alpha}\left(D_{i} f b\right)(x)$ as $k \rightarrow \infty$;

(iii) $M_{\alpha}\left(b_{h_{k}, i} f\right)(x) \rightarrow M_{\alpha}\left(D_{i} b f\right)(x)$ as $k \rightarrow \infty$.

Above facts together with 3.4 will give that

$$
\begin{aligned}
& \left|D_{i}\left(\mathfrak{M}_{b, \alpha} f\right)(x)\right| \\
= & \left|\lim _{k \rightarrow \infty}\left(\mathfrak{M}_{b, \alpha} f\right)_{h_{k}, i}(x)\right|
\end{aligned}
$$




$$
\begin{aligned}
& \leq \lim _{k \rightarrow \infty} \frac{1}{h_{k}}\left(\left|b\left(x+h_{k} e_{i}\right)\right| M_{\alpha}\left(\Delta_{h_{k} e_{i}} f\right)(x)+\left|\Delta_{h_{k} e_{i}} b(x)\right| M_{\alpha} f(x)\right. \\
& \left.+M_{\alpha}\left(\Delta_{h_{k} e_{i}} b \Delta_{h_{k} e_{i}} f\right)(x)+M_{\alpha}\left(\Delta_{h_{k} e_{i}} b f\right)(x)+M_{\alpha}\left(\Delta_{h_{k} e_{i}} f b\right)(x)\right) \\
& \leq \lim _{k \rightarrow \infty}\left(\left|b\left(x+h_{k} e_{i}\right)\right| M_{\alpha}\left(f_{h_{k}, i}\right)(x)+\left|b_{h_{k}, i}(x)\right| M_{\alpha} f(x)\right. \\
& \left.+M_{\alpha}\left(\Delta_{h_{k} e_{i}} b f_{h_{k}, i}\right)(x)+M_{\alpha}\left(b_{h_{k}, i} f\right)(x)+M_{\alpha}\left(f_{h_{k}, i} b\right)(x)\right) \\
& \leq \lim _{k \rightarrow \infty}\left(\left(\|b\|_{L i p\left(\mathbb{R}^{n}\right)}\left|h_{k}\right|+|b(x)|\right) M_{\alpha}\left(f_{h_{k}, i}\right)(x)+\left|b_{h_{k}, i}(x)\right| M_{\alpha} f(x)\right. \\
& \left.+\|b\|_{L i p\left(\mathbb{R}^{n}\right)}\left|h_{k}\right| M_{\alpha}\left(f_{h_{k}, i}\right)(x)+M_{\alpha}\left(b_{h_{k}, i} f\right)(x)+M_{\alpha}\left(f_{h_{k}, i} b\right)(x)\right) \\
& \leq|b(x)| M_{\alpha}\left(D_{i} f\right)(x)+\left|D_{i} b(x)\right| M_{\alpha} f(x)+M_{\alpha}\left(D_{i} b f\right)(x)+M_{\alpha}\left(D_{i} f b\right)(x)
\end{aligned}
$$

for all $x \in E$. This proves (3.8).

Step 3: Proof of the boundedness part. It follows from (3.7), (1.4), (1.7) and Minkowski's inequality that

$$
\begin{aligned}
\left\|\mathfrak{M}_{b, \alpha} f\right\|_{1, q} & =\left\|\mathfrak{M}_{b, \alpha} f\right\|_{q}+\left\|\nabla \mathfrak{M}_{b, \alpha} f\right\|_{q} \\
& \leq 2 A_{\alpha, n, p}\|b\|_{\infty}\|f\|_{p}+2 \sqrt{n}\|b\|_{L i p\left(\mathbb{R}^{n}\right)}\left\|M_{\alpha} f\right\|_{q}+2\|b\|_{\infty}\left\|M_{\alpha}|\nabla f|\right\|_{q} \\
& \leq 2 A_{\alpha, n, p} \sqrt{n}\|b\|_{\operatorname{Lip}\left(\mathbb{R}^{n}\right)}\|f\|_{1, p} .
\end{aligned}
$$

This finishes the proof of Theorem 1.8

\section{Proof of Theorem 1.16}

The proof of Theorem 1.16 will be divided into four steps:

$$
\text { 4.1. Proof of Theorem 1.16(i) }
$$

Let $1<p<\infty, f \in W^{1, p}(\Omega)$ and $b \in \operatorname{Lip}(\Omega)$. By Lemma 2.3 , we have that $b f \in W^{1, p}(\Omega)$ and

$$
\nabla(b f)(x)=b(x) \nabla f(x)+f(x) \nabla b(x)
$$

for almost every $x \in \Omega$. Combining (4.1) with Lemma 2.5(i) and 1.15 implies that

$$
\begin{aligned}
\left|\nabla M_{\Omega}(b f)(x)\right| & \leq 2 M_{\Omega}(\nabla(b f))(x) \leq 2 M_{\Omega}(b|\nabla f|)(x)+2 M_{\Omega}(f|\nabla b|)(x) \\
& \leq 2\|b\|_{\infty, \Omega} M_{\Omega}|\nabla f|(x)+2 \sqrt{n}\|b\|_{L i p(\Omega)} M_{\Omega} f(x)
\end{aligned}
$$

for almost every $x \in \Omega$. On the other hand, by Lemma 2.5(i), we have that $M_{\Omega} \in$ $W^{1, p}(\Omega)$ and $\left|\nabla M_{\Omega} f(x)\right| \leq 2 M_{\Omega}|\nabla f|(x)$ for almost every $x \in \Omega$. These facts together with Lemma 2.3 and 1.15 imply that

$$
\begin{aligned}
\left|\nabla\left(b M_{\Omega} f\right)(x)\right| & =\left|M_{\Omega} f(x) \nabla b(x)+b(x) \nabla M_{\Omega} f(x)\right| \\
& \leq|\nabla b(x)| M_{\Omega} f(x)|+2| b(x) \| M_{\Omega}|\nabla f|(x) \mid \\
& \leq \sqrt{n}\|b\|_{L i p(\Omega)} M_{\Omega} f(x)+2\|b\|_{\infty, \Omega} M_{\Omega}|\nabla f|(x)
\end{aligned}
$$


for almost every $x \in \Omega$. It follows from 4.2 and 4.3 that

$$
\left|\nabla\left[b, M_{\Omega}\right](f)(x)\right| \leq 4\|b\|_{\infty, \Omega} M_{\Omega}|\nabla f|(x)+3 \sqrt{n}\|b\|_{L i p(\Omega)} M_{\Omega} f(x)
$$

for almost every $x \in \Omega$. This proves (1.16). By (1.16), 1.10) and Minkowski's inequality, we have

$$
\begin{aligned}
\left\|\nabla\left[b, M_{\Omega}\right](f)\right\|_{p, \Omega} & \leq 4\|b\|_{\infty, \Omega}\left\|M_{\Omega}|\nabla f|\right\|_{p, \Omega}+3 \sqrt{n}\|b\|_{L i p(\Omega)}\left\|M_{\Omega} f\right\|_{p, \Omega} \\
& \leq C_{n, p}\left(\|b\|_{\infty, \Omega}\|\nabla f\|_{p, \Omega}+\|b\|_{L i p(\Omega)}\|f\|_{p, \Omega}\right),
\end{aligned}
$$

which together with (1.11) leads to (1.17).

It remains to prove the continuity part. Let $f_{j} \rightarrow f$ in $W^{1, p}(\Omega)$ as $j \rightarrow \infty$. By Lemma 2.3. we have that $b f_{j} \rightarrow b f$ in $W^{1, p}(\Omega)$ as $j \rightarrow \infty$. This together with Lemma 2.5(ii) implies that $M_{\Omega}\left(b f_{j}\right) \rightarrow M_{\Omega}(b f)$ in $W^{1, p}(\Omega)$ as $j \rightarrow \infty$. On the other hand, by Lemma 2.5(ii) again, one sees that $M_{\Omega} f_{j} \rightarrow M_{\Omega} f$ in $W^{1, p}(\Omega)$ as $j \rightarrow \infty$. This together with Lemma 2.3 leads to $b M_{\Omega} f_{j} \rightarrow b M_{\Omega} f$ in $W^{1, p}(\Omega)$ as $j \rightarrow \infty$. Therefore, we have that $\left[b, M_{\Omega}\right]\left(f_{j}\right) \rightarrow$ $\left[b, M_{\Omega}\right](f)$ in $W^{1, p}(\Omega)$ as $j \rightarrow \infty$.

\subsection{Proof of Theorem 1.16 (ii)}

Let $p \in(1, n), \alpha \in[1, n / p), q=n p /(n-(\alpha-1) p)$ and $q_{1}=n p /(n-\alpha p)$. It is clear that $q<q_{1}$. Let $f \in W^{1, p}(\Omega)$ and $b \in \operatorname{Lip}(\Omega)$. By Lemma 2.3, we have that $b f \in W^{1, p}(\Omega)$ and $\nabla(b f)(x)=b(x) \nabla f(x)+f(x) \nabla b(x)$ for almost every $x \in \Omega$. This together with Lemma 2.6(i) and 1.15 yields that

$$
\begin{aligned}
& \left|\nabla M_{\alpha, \Omega}(b f)(x)\right| \\
\leq & 2 M_{\alpha, \Omega}(|\nabla(b f)|)(x)+\alpha M_{\alpha-1, \Omega}(b f)(x) \\
\leq & 2 M_{\alpha, \Omega}(|\nabla b| f)(x)+2 M_{\alpha, \Omega}(|\nabla f| b)(x)+\alpha\|b\|_{\infty, \Omega} M_{\alpha-1, \Omega} f(x) \\
\leq & 2 \sqrt{n}\|b\|_{L i p(\Omega)} M_{\alpha, \Omega} f(x)+2\|b\|_{\infty, \Omega} M_{\alpha, \Omega}|\nabla f|(x)+\alpha\|b\|_{\infty, \Omega} M_{\alpha-1, \Omega} f(x)
\end{aligned}
$$

for almost every $x \in \Omega$.

On the other hand, by Lemmas 2.2, 2.6(i) and 1.15 , we have

$$
\begin{aligned}
& \left|\nabla\left(b M_{\alpha, \Omega} f\right)(x)\right| \\
\leq & |\nabla b|(x) M_{\alpha, \Omega} f(x)+|b(x)|\left|\nabla M_{\alpha, \Omega} f\right|(x) \\
\leq & \sqrt{n}\|b\|_{L i p(\Omega)} M_{\alpha, \Omega} f(x)+|b(x)|\left(2 M_{\alpha, \Omega}|\nabla f|(x)+\alpha M_{\alpha-1, \Omega} f(x)\right) \\
\leq & \left.\sqrt{n}\|b\|_{L i p(\Omega)} M_{\alpha, \Omega} f(x)+2\|b\|_{\infty, \Omega} M_{\alpha, \Omega}|\nabla f|(x)+\alpha\|b\|_{\infty, \Omega} M_{\alpha-1, \Omega} f(x)\right)
\end{aligned}
$$

for almost every $x \in \Omega$. In light of 4.4 and 4.5 we would have

$$
\begin{aligned}
& \left|\nabla\left[b, M_{\alpha, \Omega}\right](f)(x)\right| \\
\leq & \left|\nabla\left(b M_{\alpha, \Omega} f\right)(x)\right|+\left|\nabla M_{\alpha, \Omega}(b f)(x)\right| \\
\leq & 3 \sqrt{n}\|b\|_{L i p(\Omega)} M_{\alpha, \Omega} f(x)+4\|b\|_{\infty, \Omega} M_{\alpha, \Omega}|\nabla f|(x)+2 \alpha\|b\|_{\infty, \Omega} M_{\alpha-1, \Omega} f(x)
\end{aligned}
$$


for almost every $x \in \Omega$. This proves 1.18 .

By 1.18, 1.10, Minkowski's inequality and Hölder's inequality, we have

$$
\begin{aligned}
& \left\|\nabla\left[b, M_{\alpha, \Omega}\right](f)\right\|_{q, \Omega} \\
\leq & 3 \sqrt{n}\|b\|_{L i p(\Omega)}\left\|M_{\alpha, \Omega} f\right\|_{q, \Omega}+4\|b\|_{\infty, \Omega}\left\|M_{\alpha, \Omega}|\nabla f|\right\|_{q, \Omega}+2 \alpha\|b\|_{\infty, \Omega}\left\|M_{\alpha-1, \Omega} f\right\|_{q, \Omega} \\
\leq & 3 \sqrt{n}\|b\|_{L i p(\Omega)}|\Omega|^{1 / q-1 / q_{1}}\left\|M_{\alpha, \Omega} f\right\|_{q_{1}, \Omega}+4\|b\|_{\infty, \Omega}|\Omega|^{1 / q-1 / q_{1}}\left\|M_{\alpha, \Omega}|\nabla f|\right\|_{q_{1}, \Omega} \\
& +C_{\alpha, p, n}\|b\|_{\infty, \Omega}\|f\|_{p, \Omega} \\
\leq & C_{\alpha, n, p,|\Omega|}\|b\|_{\operatorname{Lip}(\Omega)}\|f\|_{1, p, \Omega},
\end{aligned}
$$

which together with (1.11) leads to (1.19).

We now prove the continuity part. Let $f_{j} \rightarrow f$ in $W^{1, p}(\Omega)$ as $j \rightarrow \infty$. By Lemma 2.3 , we see that $b f_{j} \rightarrow b f$ in $W^{1, p}(\Omega)$ as $j \rightarrow \infty$. This together with Lemma 2.6(ii) implies that $M_{\alpha, \Omega}\left(b f_{j}\right) \rightarrow M_{\alpha, \Omega}(b f)$ in $W^{1, q}(\Omega)$ as $j \rightarrow \infty$. On the other hand, by Lemma 2.6(ii), we have that $M_{\alpha, \Omega} f_{j} \rightarrow M_{\alpha, \Omega} f$ in $W^{1, q}(\Omega)$ as $j \rightarrow \infty$. This together with Lemma 2.3 leads to $b M_{\alpha, \Omega} f_{j} \rightarrow b M_{\alpha, \Omega} f$ in $W^{1, q}(\Omega)$ as $j \rightarrow \infty$. Therefore, we have that $\left[b, M_{\alpha, \Omega}\right]\left(f_{j}\right) \rightarrow$ $\left[b, M_{\alpha, \Omega}\right](f)$ in $W^{1, q}(\Omega)$ as $j \rightarrow \infty$.

\subsection{Proof of Theorem 1.16(iii)}

Let $p \in(1, n), \alpha \in[1, n / p)$ and $q=p n /(n-\alpha p)$. Set $1 / \widetilde{p}=1 / p-1 / n$. It is clear that $1 / q=1 / \widetilde{p}-(\alpha-1) / n$. By using Lemma 2.6(iii) and the arguments similar to those used to derive (1.18), one can get 1.20). By (1.20, 1.10 and Minkowski's inequality, we have

$$
\begin{aligned}
& \left\|\nabla\left[b, M_{\alpha, \Omega}\right](f)\right\|_{q, \Omega} \\
\leq & 3 \sqrt{n}\|b\|_{L i p(\Omega)}\left\|M_{\alpha, \Omega} f\right\|_{q, \Omega}+4\|b\|_{\infty, \Omega}\left\|M_{\alpha, \Omega}|\nabla f|\right\|_{q, \Omega}+2 \alpha\|b\|_{\infty, \Omega}\left\|M_{\alpha-1, \Omega} f\right\|_{q, \Omega} \\
\leq & C_{\alpha, n, p}\left(\|b\|_{L i p(\Omega)}\|f\|_{p, \Omega}+\|b\|_{\infty, \Omega}\|\nabla f\|_{p, \Omega}+\|b\|_{\infty, \Omega}\|f\|_{\tilde{p}, \Omega}\right) \\
\leq & C_{\alpha, n, p}\|b\|_{\operatorname{Lip}(\Omega)}\|f\|_{1, p, \Omega},
\end{aligned}
$$

which together with 1.11 implies 1.21 .

\subsection{Proof of Theorem 1.16(iv)}

Let $p \in(n /(n-1), \infty), \alpha \in[1, \min \{(n-1) / p, n-2 n /((n-1) p)\}+1)$ and $|\Omega|<\infty$. By Lemma 2.6(iv), we have

$$
\begin{aligned}
\left|\nabla M_{\alpha, \Omega}(b f)(x)\right| & \leq C_{n}\left(M_{\alpha-1, \Omega}(b f)(x)+\mathcal{S}_{\alpha-1, \Omega}(b f)(x)\right) \\
& \leq C_{n}\|b\|_{\infty, \Omega}\left(M_{\alpha-1, \Omega} f(x)+\mathcal{S}_{\alpha-1, \Omega} f(x)\right)
\end{aligned}
$$

for almost every $x \in \Omega$. By Lemmas 2.6(iv), 2.3 and (1.15), we have

$$
\begin{aligned}
\left|\nabla\left(b M_{\alpha, \Omega} f\right)(x)\right| & \leq|\nabla b(x)| M_{\alpha, \Omega} f(x)+|b(x)|\left|\nabla M_{\alpha, \Omega} f(x)\right| \\
& \leq \sqrt{n}\|b\|_{L i p(\Omega)} M_{\alpha, \Omega} f(x)+C_{n}\|b\|_{\infty, \Omega}\left(M_{\alpha-1, \Omega} f(x)+\mathcal{S}_{\alpha-1, \Omega} f(x)\right)
\end{aligned}
$$


for almost every $x \in \Omega$. Combining (4.6) with 4.7 implies 1.22 .

Let $1 / q_{1}=1 / p-\alpha / n$. Clearly, $q<q_{1}$. By 1.22 , (1.10), Minkowski's inequality, Hölder's inequality and Lemma 2.7, we have

$$
\begin{aligned}
& \left\|\nabla\left[b, M_{\alpha, \Omega}\right](f)\right\|_{q, \Omega} \\
\leq & \sqrt{n}\|b\|_{L i p(\Omega)}\left\|M_{\alpha, \Omega} f\right\|_{q, \Omega}+C_{n}\|b\|_{\infty, \Omega}\left(\left\|M_{\alpha-1, \Omega} f\right\|_{q, \Omega}+\left\|\mathcal{S}_{\alpha-1, \Omega} f\right\|_{q, \Omega}\right) \\
\leq & \sqrt{n}\|b\|_{L i p(\Omega)}|\Omega|^{1 / q-1 / q_{1}}\left\|M_{\alpha, \Omega} f\right\|_{q_{1}, \Omega}+C_{\alpha, n, p}\|b\|_{\infty, \Omega}\|f\|_{p, \Omega} \\
\leq & C_{\alpha, n, p,|\Omega|}\|b\|_{\operatorname{Lip}(\Omega)}\|f\|_{p, \Omega},
\end{aligned}
$$

which together with (1.11) implies 1.23 . This completes the proof of Theorem 1.16 ,

\section{Proof of Theorem 1.17}

\subsection{Preliminary notation and lemmas}

For convenience, we set $\delta(x)=\operatorname{dist}\left(x, \Omega^{c}\right)$. It is clear that $\delta$ is a Lipschitz function. By Rademacher's theorem, we see that $\delta$ is differentiable almost everywhere in $\Omega$. Moreover, $|\nabla \delta(x)|=1$ for almost every $x \in \Omega$. Let $b, f$ be two suitable functions defined on $\Omega$. For $t \in(0,1)$ and $\alpha \in(0, n)$, we define the function $A_{t, b, \alpha}(f): \Omega \rightarrow[-\infty, \infty]$ by

$$
A_{t, b, \alpha}(f)(x)=\frac{(t \delta(x))^{\alpha}}{|B(x, t \delta(x))|} \int_{B(x, t \delta(x))}|b(x)-b(y)| f(y) d y .
$$

When $\alpha=0$, we denote $A_{t, b, \alpha}=A_{t, b}$.

In what follows, for any arbitrary functions $F(x, y)$ defined on $\Omega \times \Omega$, we set $\nabla_{x} F=$ $\left(D_{1, x} F, \ldots, D_{n, x} F\right)$, where $D_{i, x} F$ is the $i$-th weak partial derivative of $F$ in $x$.

Lemma 5.1. Let $p \in(1, n), \alpha \in[1, n / p)$ and $q=n p /(n-(\alpha-1) p)$. Assume that $|\Omega|<\infty$ and $b \in \operatorname{Lip}(\Omega)$. If $f \in W^{1, p}(\Omega)$, then $A_{t, b, \alpha}(f) \in W^{1, q}(\Omega)$ and

$$
\left|\nabla A_{t, b, \alpha}(f)(x)\right| \leq \alpha \mathfrak{M}_{b, \alpha-1, \Omega} f(x)+3 \sqrt{n}\|b\|_{L i p(\Omega)} M_{\alpha, \Omega} f(x)+2 \mathfrak{M}_{b, \alpha, \Omega}|\nabla f|(x)
$$

for almost every $x \in \Omega$.

Proof. At first we assume that $f \in W^{1, p}(\Omega) \cap \mathcal{C}^{\infty}(\Omega)$. Fix $i=1,2, \ldots, n$. By $[25,(5.8)]$, we have

$$
\begin{aligned}
& \left|\nabla A_{t, b, \alpha}(f)(x)\right| \\
\leq & \alpha \frac{(t \delta(x))^{\alpha-1}}{|B(x, t \delta(x))|} \int_{B(x, t \delta(x))}|b(x)-b(y)||f(y)| d y \\
& +\frac{|\nabla \delta(x)|}{\delta(x)} \frac{(t \delta(x))^{\alpha}}{|B(x, t \delta(x))|} \int_{B(x, t \delta(x))}\left|\nabla_{y}(|b(x)-b(y)| f(y)) \cdot(y-x)\right| d y
\end{aligned}
$$




$$
\begin{aligned}
+\frac{(t \delta(x))^{\alpha}}{|B(x, t \delta(x))|} & \left(\int_{B(x, t \delta(x))}\left|\nabla_{x}\right| b(x)-b(y)|f(y)| d y\right. \\
& \left.+\int_{B(x, t \delta(x))}\left|\nabla_{y}(|b(x)-b(y)| f(y))\right| d y\right) \\
\leq \alpha \mathfrak{M}_{b, \alpha-1, \Omega} f(x) & +\frac{2(t \delta(x))^{\alpha}}{|B(x, t \delta(x))|} \int_{B(x, t \delta(x))}\left|\nabla_{y}(|b(x)-b(y)| f(y))\right| d y \\
+\frac{(t \delta(x))^{\alpha}}{|B(x, t \delta(x))|} & \int_{B(x, t \delta(x))}\left|\nabla_{x}\right| b(x)-b(y)|f(y)| d y
\end{aligned}
$$

for almost every $x \in \Omega$. Since $b \in \operatorname{Lip}(\Omega)$, then $|b(x)-b(\cdot)| \in \operatorname{Lip}(\Omega),\||b(x)-b(\cdot)|\|_{L i p(\Omega)} \leq$ $\|b\|_{\operatorname{Lip}(\Omega)}$ and $\||b(x)-b(\cdot)|\|_{\operatorname{Lip}(\Omega)} \leq 2\|b\|_{\operatorname{Lip}(\Omega)}$. Similarly, we have that $|b(\cdot)-b(y)| \in \operatorname{Lip}(\Omega)$ and $\||b(\cdot)-b(y)|\|_{L i p(\Omega)} \leq\|b\|_{L i p(\Omega)}$. Invoking Lemma 2.3 , we have that $|b(x)-b(\cdot)| f(\cdot) \in$ $W^{1, p}(\Omega)$ and

$$
\nabla_{y}(|b(x)-b(y)| f(y))=f(y) \nabla_{y}|b(x)-b(y)|+|b(x)-b(y)| \nabla f(y)
$$

for almost every $y \in \Omega$. By 1 1.15) and the fact that $\||b(x)-b(\cdot)|\|_{L i p(\Omega)} \leq\|b\|_{L i p(\Omega)}$, we have that $\left|\nabla_{y}\right| b(x)-b(y)|| \leq \sqrt{n}\|b\|_{L i p(\Omega)}$ for all $x \in \Omega$. Similarly, $\left|\nabla_{x}\right| b(x)-b(y) \| \leq$ $\sqrt{n}\|b\|_{L i p(\Omega)}$ for all $y \in \Omega$. Then we get from (5.3) that

$$
\left|\nabla_{y}(|b(x)-b(y)| f(y))\right| \leq \sqrt{n}\|b\|_{\operatorname{Lip}(\Omega)}|f(y)|+|b(x)-b(y)||\nabla f(y)|
$$

for almost every $y \in \Omega$. By (5.4), (5.2) and the fact that $\left|\nabla_{x}\right| b(x)-b(y)\|\leq \sqrt{n}\| b \|_{L i p(\Omega)}$, we have

$$
\begin{aligned}
& \left|\nabla A_{t, b, \alpha}(f)(x)\right| \\
\leq & \alpha \mathfrak{M}_{b, \alpha-1, \Omega} f(x)+\sqrt{n}\|b\|_{\operatorname{Lip}(\Omega)} \frac{(t \delta(x))^{\alpha}}{|B(x, t \delta(x))|} \int_{B(x, t \delta(x))}|f(y)| d y \\
& +\frac{2(t \delta(x))^{\alpha}}{|B(x, t \delta(x))|} \int_{B(x, t \delta(x))}\left(|b(x)-b(y)||\nabla f(y)|+\sqrt{n}\|b\|_{L i p(\Omega)}|f(y)|\right) d y \\
\leq & \alpha \mathfrak{M}_{b, \alpha-1, \Omega} f(x)+3 \sqrt{n}\|b\|_{\operatorname{Lip}(\Omega)} M_{\alpha, \Omega} f(x)+2 \mathfrak{M}_{b, \alpha, \Omega}|\nabla f|(x)
\end{aligned}
$$

for almost every $x \in \Omega$. This proves (5.1) for $f \in W^{1, p}(\Omega) \cap \mathcal{C}^{\infty}(\Omega)$.

Next we complete the rest of proof by an approximation argument. Assume that $f \in W^{1, p}(\Omega)$ for some $p \in(1, n)$. There exists a sequence of functions $\left\{\varphi_{j}\right\}_{j=1}^{\infty}$ in $W^{1, p}(\Omega) \cap$ $\mathcal{C}^{\infty}(\Omega)$ such that $\varphi_{j} \rightarrow f$ in $W^{1, p}(\Omega)$ as $j \rightarrow \infty$. By Hölder's inequality, we have

$$
\begin{aligned}
\left|A_{t, b, \alpha}\left(\varphi_{j}\right)(x)-A_{t, b, \alpha}(f)(x)\right| & \leq \frac{(t \delta(x))^{\alpha}}{|B(x, t \delta(x))|} \int_{B(x, t \delta(x))}|b(x)-b(y)|\left|\varphi_{j}(y)-f(y)\right| d y \\
& \leq\left(|b(x)|+\|b\|_{\infty, \Omega}\right)\left\|\varphi_{j}-f\right\|_{p, \Omega} \frac{(t \delta(x))^{\alpha}}{|B(x, t \delta(x))|^{1 / p}},
\end{aligned}
$$


which leads to

$$
\lim _{j \rightarrow \infty} A_{t, b, \alpha}\left(\varphi_{j}\right)(x)=A_{t, b, \alpha}(f)(x)
$$

for all $x \in \Omega$. It was shown that

$$
\left|\nabla A_{t, b, \alpha}\left(\varphi_{j}\right)(x)\right| \leq \alpha \mathfrak{M}_{b, \alpha-1, \Omega} \varphi_{j}(x)+3 \sqrt{n}\|b\|_{L i p(\Omega)} M_{\alpha, \Omega} \varphi_{j}(x)+2 \mathfrak{M}_{b, \alpha, \Omega}\left|\nabla \varphi_{j}\right|(x)
$$

for almost every $x \in \Omega$. Let $q_{1}=n p /(n-\alpha p)$. It is clear that $q<q_{1}$. By 1.15, (1.13), (5.6), Hölder's inequality and Minkowski's inequality, we have

$$
\begin{aligned}
& \left\|\nabla A_{t, b, \alpha}\left(\varphi_{j}\right)\right\|_{q, \Omega} \\
\leq & \alpha\left\|\mathfrak{M}_{b, \alpha-1, \Omega} \varphi_{j}\right\|_{q, \Omega}+3 \sqrt{n}\|b\|_{L i p(\Omega)}\left\|M_{\alpha, \Omega} \varphi_{j}\right\|_{q, \Omega}+2\left\|\mathfrak{M}_{b, \alpha, \Omega}\left|\nabla \varphi_{j}\right|\right\|_{q, \Omega} \\
\leq & C_{\alpha, n, p}\|b\|_{\infty, \Omega}\left\|\varphi_{j}\right\|_{p, \Omega}+3 \sqrt{n}\|b\|_{L i p(\Omega)}|\Omega|^{1 / q-1 / q_{1}}\left\|M_{\alpha, \Omega} \varphi_{j}\right\|_{q_{1}, \Omega} \\
& +2|\Omega|^{1 / q-1 / q_{1}}\left\|\mathfrak{M}_{b, \alpha, \Omega}\left|\nabla \varphi_{j}\right|\right\|_{q_{1}, \Omega} \\
\leq & C_{\alpha, n, p,|\Omega|}\|b\|_{\operatorname{Lip}(\Omega)}\left\|\varphi_{j}\right\|_{1, p, \Omega} .
\end{aligned}
$$

This yields that $\left\{\mid \nabla A_{t, b, \alpha}\left(\varphi_{j}\right)\right\}_{j=1}^{\infty}$ is a bounded sequence in $L^{q}(\Omega)$. By the fact that $A_{t, b, \alpha}\left(\varphi_{j}\right)(x) \rightarrow A_{t, b, \alpha}(f)(x)$ as $j \rightarrow \infty$ for almost every $x \in \Omega$, we have that the Sobolev derivative $\nabla A_{t, b, \alpha}(f)$ exists almost everywhere in $\Omega$ and there exists a subsequence $\left\{\nabla A_{t, b, \alpha}\left(\varphi_{j \ell}\right)\right\}_{\ell=1}^{\infty}$ of $\left\{\nabla A_{t, b, \alpha}\left(\varphi_{j}\right)\right\}_{j=1}^{\infty}$ such that

$$
\nabla A_{t, b, \alpha}\left(\varphi_{j_{\ell}}\right) \rightarrow \nabla A_{t, b, \alpha}(f) \quad \text { weakly in } L^{q}(\Omega) \text { as } \ell \rightarrow \infty
$$

On the other hand, we get by Remark 1.11 (iii) that $\mathfrak{M}_{b, \alpha-1, \Omega} \varphi_{j} \rightarrow \mathfrak{M}_{b, \alpha-1, \Omega} f$ in $L^{q}(\Omega)$ and $\mathfrak{M}_{b, \alpha, \Omega}\left|\nabla \varphi_{j}\right| \rightarrow \mathfrak{M}_{b, \alpha, \Omega}|\nabla f|$ and $M_{\alpha, \Omega} \varphi_{j} \rightarrow M_{\alpha, \Omega} f$ in $L^{q_{1}}(\Omega)$ as $j \rightarrow \infty$. Hence, by Hölder's inequality, we have that $\mathfrak{M}_{b, \alpha, \Omega}\left|\nabla \varphi_{j}\right| \rightarrow \mathfrak{M}_{b, \alpha, \Omega}|\nabla f|$ and $M_{\alpha, \Omega} \varphi_{j} \rightarrow M_{\alpha, \Omega} f$ in $L^{q}(\Omega)$ as $j \rightarrow \infty$. For convenience, we set

$$
\mathfrak{h}_{\ell}:=\alpha \mathfrak{M}_{b, \alpha-1, \Omega} \varphi_{j_{\ell}}(x)+3 \sqrt{n}\|b\|_{L i p(\Omega)} M_{\alpha, \Omega} \varphi_{j_{\ell}}(x)+2 \mathfrak{M}_{b, \alpha, \Omega}\left|\nabla \varphi_{j_{\ell}}\right|(x)
$$

It was proved that

$$
\mathfrak{h}_{\ell} \rightarrow \alpha \mathfrak{M}_{b, \alpha-1, \Omega} f+3 \sqrt{n}\|b\|_{L i p(\Omega)} M_{\alpha, \Omega} f+2 \mathfrak{M}_{b, \alpha, \Omega}|\nabla f| \quad \text { in } L^{q}(\Omega) \text { as } \ell \rightarrow \infty
$$

Combining (5.9) with (5.6), (5.8) and Proposition 2.2 yields 5.1).

Lemma 5.2. Let $1<p<\infty$ and $b \in \operatorname{Lip}(\Omega)$. If $f \in W^{1, p}(\Omega)$, then $A_{t, b}(f) \in W^{1, p}(\Omega)$ and

$$
\left|\nabla A_{t, b}(f)(x)\right| \leq 3 \sqrt{n}\|b\|_{L i p(\Omega)} M_{\Omega} f(x)+2 \mathfrak{M}_{b, \Omega}|\nabla f|(x)
$$

for almost every $x \in \Omega$. 
Proof. At first we assume that $f \in W^{1, p}(\Omega) \cap \mathcal{C}^{\infty}(\Omega)$. By 5.2 and the arguments similar to those used in deriving 5.5 ,

$$
\left|\nabla A_{t, b}(f)(x)\right| \leq 3 \sqrt{n}\|b\|_{L i p(\Omega)} M_{\Omega} f(x)+2 \mathfrak{M}_{b, \Omega}|\nabla f|(x)
$$

for almost every $x \in \Omega$. This proves 5.10 for the case $f \in W^{1, p}(\Omega) \cap \mathcal{C}^{\infty}(\Omega)$. The rest of the proof follows from the arguments similar to those used in the proof of Lemma 5.1 . We omit the details.

Lemma 5.3. Let $p \in(1, n), \alpha \in[1, n / p)$ and $q=p n /(n-\alpha p)$. Let $\Omega$ admit a $p$-Sobolev embedding and $b \in \operatorname{Lip}(\Omega)$. If $f \in W^{1, p}(\Omega)$, then $A_{t, b, \alpha}(f) \in W^{1, q}(\Omega)$ and

$$
\left|\nabla A_{t, b, \alpha}(f)(x)\right| \leq \alpha \mathfrak{M}_{b, \alpha-1, \Omega} f(x)+2 \mathfrak{M}_{b, \alpha, \Omega}|\nabla f|(x)+3 \sqrt{n}\|b\|_{L i p(\Omega)} M_{\alpha, \Omega} f(x)
$$

for almost every $x \in \Omega$.

Proof. By (5.5), we known that (5.11) holds for all $f \in W^{1, p}(\Omega) \cap \mathcal{C}^{\infty}(\Omega)$. The rest of the proof follows from an approximation argument. Assume that $f \in W^{1, p}(\Omega)$ for some $p \in(1, n)$. There exists a sequence of functions $\left\{\varphi_{j}\right\}_{j=1}^{\infty}$ in $W^{1, p}(\Omega) \cap \mathcal{C}^{\infty}(\Omega)$ such that $\varphi_{j} \rightarrow f$ in $W^{1, p}(\Omega)$ as $j \rightarrow \infty$. It was known that

$$
\lim _{j \rightarrow \infty} A_{t, b, \alpha}\left(\varphi_{j}\right)(x)=A_{t, b, \alpha}(f)(x)
$$

for all $x \in \Omega$ and

$$
\left|\nabla A_{t, b, \alpha}\left(\varphi_{j}\right)(x)\right| \leq \alpha \mathfrak{M}_{b, \alpha-1, \Omega} \varphi_{j}(x)+3 \sqrt{n}\|b\|_{L i p(\Omega)} M_{\alpha, \Omega} \varphi_{j}(x)+2 \mathfrak{M}_{b, \alpha, \Omega}\left|\nabla \varphi_{j}\right|(x)
$$

for almost every $x \in \Omega$.

Let $1 / \widetilde{p}=1 / p-1 / n$. Clearly, $1 / q=1 / \widetilde{p}-(\alpha-1) / n$. Since $\Omega$ admits a $p$-Sobolev embedding, then

$$
\|u\|_{\widetilde{p}, \Omega} \leq C_{p, n}\|u\|_{1, p, \Omega}, \quad \forall u \in L^{p}(\Omega) .
$$

By (1.10), 1.13), 5.12, (5.13) and Minkowski's inequality, we have

$$
\begin{aligned}
& \left\|\nabla A_{t, b, \alpha}\left(\varphi_{j}\right)\right\|_{q, \Omega} \\
\leq & \alpha\left\|\mathfrak{M}_{b, \alpha-1, \Omega} \varphi_{j}\right\|_{q, \Omega}+3 \sqrt{n}\|b\|_{L i p(\Omega)}\left\|M_{\alpha, \Omega} \varphi_{j}\right\|_{q, \Omega}+2\left\|\mathfrak{M}_{b, \alpha, \Omega}\left|\nabla \varphi_{j}\right|\right\|_{q, \Omega} \\
\leq & C_{\alpha, n, p}\|b\|_{\infty, \Omega}\left(\left\|\varphi_{j}\right\|_{\tilde{p}, \Omega}+\left\|\nabla \varphi_{j}\right\|_{p, \Omega}\right)+C_{\alpha, n, p}\|b\|_{L i p(\Omega)}\left\|\varphi_{j}\right\|_{p, \Omega} \\
\leq & C_{\alpha, n, p}\|b\|_{\operatorname{Lip}(\Omega)}\left\|\varphi_{j}\right\|_{1, p, \Omega} .
\end{aligned}
$$

On the other hand, we get by $(5.13)$ that $\varphi_{j} \rightarrow f$ in $L^{\widetilde{p}}(\Omega)$ as $j \rightarrow \infty$. Then by Remark 1.11 , we have that $\mathfrak{M}_{b, \alpha, \Omega}\left|\nabla \varphi_{j}\right| \rightarrow \mathfrak{M}_{b, \alpha, \Omega}|\nabla f|, M_{\alpha, \Omega} \varphi_{j} \rightarrow M_{\alpha, \Omega} f$ and $\mathfrak{M}_{b, \alpha-1, \Omega} \varphi_{j} \rightarrow$ $\mathfrak{M}_{b, \alpha-1, \Omega} f$ in $L^{q}(\Omega)$ as $j \rightarrow \infty$. The rest of the proof follows from the arguments similar to those used in the proof of Lemma 5.1. We omit the details. 
Lemma 5.4. Let $p \in(n /(n-1), \infty), \alpha \in[1, \min \{(n-1) / p, n-2 n /((n-1) p)\}+1)$, $q=n p /(n-(\alpha-1) p)$ and $|\Omega|<\infty$. If $b \in \operatorname{Lip}(\Omega)$ and $f \in L^{p_{1}}(\Omega)$, then $A_{t, b}(f) \in W^{1, q}(\Omega)$ and

$$
\begin{aligned}
\left|\nabla A_{t, b, \alpha}(f)(x)\right| \leq & (n-\alpha) \mathfrak{M}_{b, \alpha-1, \Omega} f(x)+\sqrt{n}\|b\|_{L i p(\Omega)} M_{\alpha, \Omega} f(x) \\
& +2 n\|b\|_{\infty, \Omega} \mathcal{S}_{\alpha-1, \Omega} f(x)
\end{aligned}
$$

for almost every $x \in \Omega$.

Proof. Let $f \in L^{p}(\Omega) \cap \mathcal{C}^{\infty}(\Omega)$. It was shown in the proof of [25, Lemma 5.3] that

$$
\begin{aligned}
\left|\nabla A_{t, b, \alpha}(f)(x)\right| \leq(n-\alpha) \frac{|\nabla \delta(x)|}{|\delta(x)|} & \frac{(t \delta(x))^{\alpha}}{|B(x, t \delta(x))|} \int_{B(x, t \delta(x))}|b(x)-b(y)||f(y)| d y \\
+\frac{(t \delta(x))^{\alpha}}{|B(x, t \delta(x))|} & \left(\int_{B(x, t \delta(x))}\left|\nabla_{x}\right| b(x)-b(y)|||f(y)| d y\right. \\
& +\int_{\partial B(x, t \delta(x))}|b(x)-b(y)||f(y)||\nu(y)| d \mathcal{H}^{n-1}(y) \\
& \left.+t \int_{\partial B(x, t \delta(x))}|b(x)-b(y)||f(y)| d \mathcal{H}^{n-1}(y)|\nabla \delta(x)|\right)
\end{aligned}
$$

for almost every $x \in \Omega$, where $\nu(y)=(y-x) /(t \delta(x))$. This together with the fact that $\left|\nabla_{x}\right| b(x)-b(y)\|\leq \sqrt{n}\| b \|_{\operatorname{Lip}(\Omega)}$ implies that

$$
\begin{aligned}
& \quad\left|\nabla A_{t, b}(f)(x)\right| \\
& \leq(n-\alpha) \frac{(t \delta(x))^{\alpha-1}}{|B(x, t \delta(x))|} \int_{B(x, t \delta(x))}|b(x)-b(y)||f(y)| d y \\
& \quad+\sqrt{n}\|b\|_{L i p(\Omega)} \frac{(t \delta(x))^{\alpha}}{|B(x, t \delta(x))|} \int_{B(x, t \delta(x))}|f(y)| d y \\
& \quad+\frac{n(t \delta(x))^{\alpha-1}}{|\partial B(x, t \delta(x))|} \int_{\partial B(x, t \delta(x))}|b(x)-b(y) \| f(y)| d \mathcal{H}^{n-1}(y) \\
& \leq \\
& \quad(n-\alpha) \mathfrak{M}_{b, \alpha-1, \Omega} f(x)+\sqrt{n}\|b\|_{\operatorname{Lip}(\Omega)} M_{\alpha, \Omega} f(x)+2 n\|b\|_{\infty, \Omega} \mathcal{S}_{\alpha-1, \Omega} f(x)
\end{aligned}
$$

for almost every $x \in \Omega$.

The rest of the proof follows an approximation argument. Assume that $f \in L^{p}(\Omega)$ for some $p \in(1, n)$. There exists a sequence of functions $\left\{\varphi_{j}\right\}_{j=1}^{\infty}$ in $L^{p}(\Omega) \cap \mathcal{C}^{\infty}(\Omega)$ such that $\varphi_{j} \rightarrow f$ in $L^{p}(\Omega)$ as $j \rightarrow \infty$. It was known that

$$
\lim _{j \rightarrow \infty} A_{t, b, \alpha}\left(\varphi_{j}\right)=A_{t, b, \alpha}(f)(x)
$$

for all $x \in \Omega$. Moreover, it was proved that

$$
\begin{aligned}
\left|\nabla A_{t, b, \alpha}\left(\varphi_{j}\right)(x)\right| \leq & (n-\alpha) \mathfrak{M}_{b, \alpha-1, \Omega} \varphi_{j}(x)+\sqrt{n}\|b\|_{L i p(\Omega)} M_{\alpha, \Omega} \varphi_{j}(x) \\
& +2 n\|b\|_{\infty, \Omega} \mathcal{S}_{\alpha-1, \Omega} \varphi_{j}(x)
\end{aligned}
$$


for almost every $x \in \Omega$. Let $q_{1}=n p /(n-\alpha p)$. Clearly, $q<q_{1}$. By (1.10), 1.13), (5.15), Minkowski's inequality, Hölder's inequality and Lemma 2.7, we have

$$
\begin{aligned}
& \left\|\nabla A_{t, b, \alpha}\left(\varphi_{j}\right)\right\|_{q, \Omega} \\
\leq & (n-\alpha)\left\|\mathfrak{M}_{b, \alpha-1, \Omega} \varphi_{j}\right\|_{q, \Omega}+\sqrt{n}\|b\|_{L i p(\Omega)}\left\|M_{\alpha, \Omega} \varphi_{j}\right\|_{q, \Omega}+2 n\|b\|_{\infty, \Omega}\left\|\mathcal{S}_{\alpha-1, \Omega} \varphi_{j}\right\|_{q, \Omega} \\
\leq & C_{\alpha, n, p}\|b\|_{\infty, \Omega}\left\|\varphi_{j}\right\|_{p, \Omega}+\sqrt{n}\|b\|_{L i p(\Omega)}|\Omega|^{1 / q-1 / q_{1}}\left\|M_{\alpha, \Omega} \varphi_{j}\right\|_{q_{1}, \Omega} \\
& +C_{\alpha, n, p}\|b\|_{\infty, \Omega}\left\|\varphi_{j}\right\|_{p, \Omega} \\
\leq & C_{\alpha, n, p,|\Omega|}\|b\|_{\operatorname{Lip}(\Omega)}\left\|\varphi_{j}\right\|_{p, \Omega} .
\end{aligned}
$$

On the other hand, by Remark 1.11, we have that $\mathfrak{M}_{b, \alpha-1, \Omega} \varphi_{j} \rightarrow \mathfrak{M}_{b, \alpha-1, \Omega} f$ in $L^{q}(\Omega)$ as $j \rightarrow \infty$. Moreover, $M_{\alpha, \Omega} \varphi_{j} \rightarrow M_{\alpha, \Omega} f$ in $L^{q_{1}}(\Omega)$ as $j \rightarrow \infty$. This together with Hölder's inequality implies that $M_{\alpha, \Omega} \varphi_{j} \rightarrow M_{\alpha, \Omega} f$ in $L^{q}(\Omega)$ as $j \rightarrow \infty$. By the sublinearity and Lemma 2.7, one sees that $\mathcal{S}_{\alpha-1, \Omega} \varphi_{j} \rightarrow \mathcal{S}_{\alpha-1, \Omega} f$ in $L^{q}(\Omega)$ as $j \rightarrow \infty$. The rest of the proof follows from the arguments similar to those used in the proof of Lemma 5.1. We omit the details.

\subsection{Proof of Theorem 1.17}

We adopt the method of 17 to prove Theorem 1.17. Let $t_{j}, j=1,2, \ldots$, be an enumeration of the rationals between 0 and 1 . For any $k \geq 1,0 \leq \alpha<n$ and two suitable functions $f$, $b$ defined on $\Omega$, we define the operator $u_{k, b, \alpha}$ by

$$
u_{k, b, \alpha}(f)(x)=\max _{1 \leq j \leq k} A_{t_{j}, b, \alpha}(f)(x) .
$$

For $\alpha=0$, we denote $u_{k, b, \alpha}=u_{k, b}$.

We first prove (i). Let $f \in W^{1, p}(\Omega)$ with $p \in(1, \infty)$ and $b \in \operatorname{Lip}(\Omega)$. Invoking Lemma 5.2 , one has that $A_{t_{j}, b}(f) \in W^{1, p}(\Omega)$ and

$$
\left|\nabla A_{t_{j}, b}(f)(x)\right| \leq 3 \sqrt{n}\|b\|_{L i p(\Omega)} M_{\Omega} f(x)+2 \mathfrak{M}_{b, \Omega}|\nabla f|(x)
$$

for all $j=1,2, \ldots$ and almost every $x \in \Omega$. On the other hand, it is easy to see that

$$
\mathfrak{M}_{b, \Omega} f(x)=\sup _{j \geq 1} A_{t_{j}, b}(f)(x)
$$

for all $x \in \Omega$. Moreover, the sequence $\left\{u_{k, b}\right\}_{k=1}^{\infty}$ is an increasing sequence of functions converging pointwise to $\mathfrak{M}_{b, \Omega} f$. Using (5.17) and the fact that the maximum of two Sobolev functions belongs to the Sobolev space (see [11, Lemma 7.6]), one finds that

$$
\begin{aligned}
\left|\nabla u_{k, b}(x)\right| & =\left|\nabla \max _{1 \leq j \leq k} A_{t_{j}, b}(f)(x)\right| \leq \max _{1 \leq j \leq k}\left|\nabla A_{t_{j}, b}(f)(x)\right| \\
& \leq 3 \sqrt{n}\|b\|_{\operatorname{Lip}(\Omega)} M_{\Omega} f(x)+2 \mathfrak{M}_{b, \Omega}|\nabla f|(x)
\end{aligned}
$$


for all $k=1,2, \ldots$ and almost every $x \in \Omega$. By (5.18, 1.10, (1.13) and Minkowski's inequality, we have

$$
\begin{aligned}
\left\|\nabla u_{k, b}\right\|_{p, \Omega} & \leq 3 \sqrt{n}\|b\|_{\operatorname{Lip}(\Omega)}\left\|M_{\Omega} f\right\|_{p, \Omega}+2\left\|\mathfrak{M}_{b, \Omega}|\nabla f|\right\|_{p, \Omega} \\
& \leq C_{n, p}\left(\|b\|_{\operatorname{Lip}(\Omega)}\|f\|_{p, \Omega}+\|b\|_{\infty, \Omega}\|\nabla f\|_{p, \Omega}\right) \leq C_{n, p}\|b\|_{\operatorname{Lip}(\Omega)}\|f\|_{1, p, \Omega},
\end{aligned}
$$

which gives that $\left\{\left|\nabla u_{k, b}\right|\right\}_{k=1}^{\infty}$ is a bounded sequence in $L^{p}(\Omega)$. Since $u_{k, b}$ converges pointwise to $\mathfrak{M}_{b, \Omega} f$ as $k \rightarrow \infty$, then the weak gradient $\nabla \mathfrak{M}_{b, \Omega} f$ exists and there exists a subsequence $\left\{u_{k_{\ell}, b}\right\}_{\ell=1}^{\infty}$ of $\left\{u_{k, b}\right\}_{k=1}^{\infty}$ such that $\left|\nabla u_{k_{\ell}, b}\right| \rightarrow\left|\nabla \mathfrak{M}_{b, \Omega} f\right|$ weakly in $L^{p}(\Omega)$ as $\ell \rightarrow \infty$. The estimate (1.24) follows from the same argument as in the end of the proof of Lemma 5.1.

By (1.24) and the arguments similar to those used to derive (5.19), we have

$$
\left\|\nabla \mathfrak{M}_{b, \Omega} f\right\|_{p, \Omega} \leq C_{n, p}\|b\|_{\operatorname{Lip}(\Omega)}\|f\|_{1, p, \Omega},
$$

which combining with (1.14 implies that

$$
\left\|\mathfrak{M}_{b, \Omega} f\right\|_{1, p, \Omega}=\left\|\mathfrak{M}_{b, \Omega} f\right\|_{p, \Omega}+\left\|\nabla \mathfrak{M}_{b, \Omega} f\right\|_{p, \Omega} \leq C_{n, p}\|b\|_{\operatorname{Lip}(\Omega)}\|f\|_{1, p, \Omega},
$$

which proves 1.25 .

Using Lemma 5.1 and the arguments similar to those used in deriving (1.24), we can prove (1.26). By 1.26 and the arguments similar to those used to derive (5.7),

$$
\left\|\nabla \mathfrak{M}_{b, \alpha, \Omega} f\right\|_{q, \Omega} \leq C_{\alpha, n, p,|\Omega|}\|b\|_{\operatorname{Lip}(\Omega)}\|f\|_{1, p, \Omega},
$$

which together with 1.13 leads to 1.27).

By Lemma 5.3 and the arguments similar to those used in deriving (1.24), we can prove (1.28). By (1.28) and the arguments similar to those used to derive (5.14),

$$
\left\|\nabla \mathfrak{M}_{b, \alpha, \Omega} f\right\|_{q, \Omega} \leq C_{\alpha, n, p}\|b\|_{\operatorname{Lip}(\Omega)}\|f\|_{1, p, \Omega},
$$

which together with 1.13 leads to 1.29 .

Finally, using Lemma 5.4 and the arguments similar to those used in deriving (1.24), we can prove (1.30). By 1.30 and the arguments similar to those used to derive (5.16),

$$
\left\|\nabla \mathfrak{M}_{b, \alpha, \Omega} f\right\|_{q, \Omega} \leq C_{\alpha, n, p,|\Omega|}\|b\|_{\operatorname{Lip}(\Omega)}\|f\|_{p, \Omega},
$$

which together with (1.13) leads to (1.31). This completes the proof of Theorem 1.17 .

$$
\text { 6. Proof of Corollary } 1.18
$$

To prove Corollary 1.18, we need the following property of the Sobolev space with zero boundary values. 
Lemma 6.1. [18 Let $\Omega \subset \mathbb{R}^{n}, \Omega \neq \mathbb{R}^{n}$, be an open set. Let $f \in W^{1, p}(\Omega)$ for $1<p<\infty$ and $\int_{\Omega}\left(\frac{f(x)}{\operatorname{dist}\left(x, \Omega^{c}\right)}\right)^{p} d x<\infty$. Then $f \in W_{0}^{1, p}(\Omega)$.

Proof of Corollary 1.18. The proof of Corollary 1.18 will be divided into four steps:

(i) Let $f \in W_{0}^{1, p}(\Omega)$ with $p \in(1, \infty)$ and $b \in \operatorname{Lip}(\Omega)$. There exists a sequence of functions $\left\{\varphi_{k}\right\}_{k=1}^{\infty}$ in $\mathcal{C}_{0}^{\infty}(\Omega)$ such that $\varphi_{k} \rightarrow f$ in $W^{1, p}(\Omega)$ as $k \rightarrow \infty$. It follows from Theorem 1.16(i) that $\left[b, M_{\Omega}\right]\left(\varphi_{k}\right) \in W^{1, p}(\Omega), k=1,2, \ldots$ Note that $\left[b, M_{\Omega}\right]\left(\varphi_{k}\right)(x)=0$ whenever $\operatorname{dist}(x, \partial \Omega)<1 /\left(2 \operatorname{dist}\left(\operatorname{supp} \varphi_{k}, \partial \Omega\right)\right)$. Thus we have $\left[b, M_{\Omega}\right]\left(\varphi_{k}\right) \in W_{0}^{1, p}(\Omega)$. By Remark 1.11(ii) we see that $\left[b, M_{\Omega}\right]\left(\varphi_{k}\right) \rightarrow\left[b, M_{\Omega}\right](f)$ in $L^{p}(\Omega)$ as $k \rightarrow \infty$. On the other hand, by Theorem $1.16(\mathrm{i})$,

$$
\left|\nabla\left[b, M_{\Omega}\right]\left(\varphi_{k}\right)(x)\right| \leq 4\|b\|_{\infty, \Omega} M_{\Omega}\left|\nabla \varphi_{k}\right|(x)+3 \sqrt{n}\|b\|_{L i p(\Omega)} M_{\Omega} \varphi_{k}(x)
$$

for almost every $x \in \Omega$. This together with the arguments similar to those used to derive (1.17) implies

$$
\left\|\left[b, M_{\Omega}\right]\left(\varphi_{k}\right)\right\|_{1, p, \Omega} \leq C_{n, p}\|b\|_{\operatorname{Lip}(\Omega)}\left\|\varphi_{k}\right\|_{1, p, \Omega} .
$$

This yields that $\left\{\left[b, M_{\Omega}\right]\left(\varphi_{k}\right)\right\}$ is a bounded sequence in $W_{0}^{1, p}(\Omega)$ converging to $\left[b, M_{\Omega}\right](f)$ in $L^{p}(\Omega)$. A weak compactness implies $\left[b, M_{\Omega}\right](f) \in W_{0}^{1, p}(\Omega)$. Similarly, we can prove $\mathfrak{M}_{b, \Omega} f \in W_{0}^{1, p}(\Omega)$.

(ii) Let $f \in W^{1, p}(\Omega)$ with $p \in(1, n)$ and $\alpha, q, \Omega$ be given as in Corollary 1.18 (ii). It is easy to see that $M_{\alpha, \Omega} f(x) \leq \operatorname{dist}\left(x, \Omega^{c}\right) M_{\alpha-1, \Omega} f(x)$ for any $x \in \Omega$. It follows that

$$
\begin{aligned}
\left|\left[b, M_{\alpha, \Omega}\right](f)(x)\right| & \leq \operatorname{dist}\left(x, \Omega^{c}\right)\left(|b(x)| M_{\alpha-1, \Omega} f(x)+M_{\alpha-1, \Omega}(b f)(x)\right) \\
& \leq 2\|b\|_{\infty, \Omega} \operatorname{dist}\left(x, \Omega^{c}\right) M_{\alpha-1, \Omega} f(x)
\end{aligned}
$$

for all $x \in \Omega$. In light of $(6.1)$ and 1.10$)$ we would have

$$
\begin{aligned}
\left(\int_{\Omega}\left(\frac{\left[b, M_{\alpha, \Omega}\right](f)(x)}{\operatorname{dist}\left(x, \Omega^{c}\right)}\right)^{q} d x\right)^{1 / q} & \leq 2\|b\|_{\infty, \Omega}\left\|M_{\alpha-1, \Omega} f\right\|_{q, \Omega} \\
& \leq C_{\alpha, n, p}\|b\|_{\infty, \Omega}\|f\|_{p, \Omega}<\infty
\end{aligned}
$$

On the other hand, we get from Theorem 1.16 (ii) that $\left[b, M_{\alpha, \Omega}\right](f) \in W^{1, q}(\Omega)$. This together with 6.2 and Lemma 6.1 yields $\left[b, M_{\alpha, \Omega}\right](f) \in W_{0}^{1, q}(\Omega)$.

One can easily check that

$$
\mathfrak{M}_{b, \alpha, \Omega} f(x) \leq 2\|b\|_{\infty, \Omega} M_{\alpha, \Omega} f(x) \leq 2\|b\|_{\infty, \Omega} \operatorname{dist}\left(x, \Omega^{c}\right) M_{\alpha-1, \Omega} f(x)
$$

which together with Theorem 1.17(ii) and the arguments similar to those used in deriving $\left[b, M_{\alpha, \Omega}\right](f) \in W_{0}^{1, q}(\Omega)$ implies $\mathfrak{M}_{b, \alpha, \Omega} f \in W_{0}^{1, q}(\Omega)$. 
(iii) Let $f \in W^{1, p}(\Omega)$ and $\alpha, p, q, \Omega$ be given as in Corollary 1.18 (iii). Let $1 / \widetilde{p}=$ $1 / p-1 / n$. Clearly, $1 / q=1 / \widetilde{p}-(\alpha-1) / n$. By (6.1), 1.10) and the $p$-Sobolev embedding property of $\Omega$, we have

$$
\begin{aligned}
\left(\int_{\Omega}\left(\frac{\left[b, M_{\alpha, \Omega}\right](f)(x)}{\operatorname{dist}\left(x, \Omega^{c}\right)}\right)^{q} d x\right)^{1 / q} & \leq 2\|b\|_{\infty, \Omega}\left\|M_{\alpha-1, \Omega} f\right\|_{q, \Omega} \leq C_{\alpha, n, p}\|b\|_{\infty, \Omega}\|f\|_{\widetilde{p}, \Omega} \\
& \leq C_{\alpha, n, p}\|b\|_{\infty, \Omega}\|f\|_{1, p, \Omega}<\infty
\end{aligned}
$$

On the other hand, we get from Theorem 1.16(iii) that $\left[b, M_{\alpha, \Omega}\right](f) \in W^{1, q}(\Omega)$. This together with (6.4) and Lemma 6.1 yields $\left[b, M_{\alpha, \Omega}\right](f) \in W_{0}^{1, q}(\Omega)$. Similarly, we get by (6.3) and Theorem 1.17 (iii) that $\mathfrak{M}_{b, \alpha, \Omega} f \in W_{0}^{1, q}(\Omega)$.

(iv) Let $f \in L^{p}(\Omega)$ and $p, \alpha, q$ be given as in Corollary 1.18(iv). By (6.2), we have

$$
\left(\int_{\Omega}\left(\frac{\left[b, M_{\alpha, \Omega}\right](f)(x)}{\operatorname{dist}\left(x, \Omega^{c}\right)}\right)^{q} d x\right)^{1 / q} \leq C_{\alpha, n, p}\|f\|_{p, \Omega}<\infty .
$$

By Theorem 1.16(iv) we have $\left[b, M_{\alpha, \Omega}\right](f) \in W^{1, q}(\Omega)$. This together with 6.5 and Lemma 6.1 yields $\left[b, M_{\alpha, \Omega}\right](f) \in W_{0}^{1, q}(\Omega)$.

On the other hand, by 6.3 and the arguments similar to those used to derive 6.2 ,

$$
\left(\int_{\Omega}\left(\frac{\mathfrak{M}_{b, \alpha, \Omega} f(x)}{\operatorname{dist}\left(x, \Omega^{c}\right)}\right)^{q} d x\right)^{1 / q} \leq C_{\alpha, n, p}\|f\|_{p, \Omega}<\infty .
$$

This together with Theorem 1.17 (iv) and Lemma 6.1 implies $\mathfrak{M}_{b, \alpha, \Omega} f \in W_{0}^{1, q}(\Omega)$.

\section{Acknowledgments}

This work was supported partly by NNSF of China (No. 11701333). The authors want to express their sincerely thanks to the referees for their valuable remarks and suggestions, which made this paper more readable.

\section{References}

[1] M. Agcayazi, A. Gogatishvili, K. Koca and R. Mustafayev, A note on maximal commutators and commutators of maximal functions, J. Math. Soc. Japan 67 (2015), no. 2, 581-593.

[2] J. M. Aldaz and J. Pérez Lázaro, Functions of bounded variation, the derivative of the one dimensional maximal function, and applications to inequalities, Trans. Amer. Math. Soc. 359 (2007), no. 5, 2443-2461.

[3] J. Bastero, M. Milman and F. J. Ruiz, Commutators for the maximal and sharp functions, Proc. Amer. Math. Soc. 128 (2000), no. 11, 3329-3334. 
[4] A. Bonami, T. Iwaniec, P. Jones and M. Zinsmeister, On the product of functions in BMO and $H^{1}$, Ann. Inst. Fourier (Grenoble) 57 (2007), no. 5, 1405-1439.

[5] E. Carneiro and J. Madrid, Derivative bounds for fractional maximal functions, Trans. Amer. Math. Soc. 369 (2017), no. 6, 4063-4092.

[6] E. Carneiro, J. Madrid and L. B. Pierce, Endpoint Sobolev and BV continuity for maximal operators, J. Funct. Anal. 273 (2017), no. 10, 3262-3294.

[7] E. Carneiro and D. Moreira, On the regularity of maximal operators, Proc. Amer. Math. Soc. 136 (2008), no. 12, 4395-4404.

[8] E. Carneiro and B. F. Svaiter, On the variation of maximal operators of convolution type, J. Funct. Anal. 265 (2013), no. 5, 837-865.

[9] F. Deringoz, V. S. Guliyev and S. G. Hasanov, Commutators of fractional maximal operator on generalized Orlicz-Morrey spaces, Positivity 22 (2018), no. 1, 141-158.

[10] J. García-Cuerva, E. Harboure, C. Segovia and J. L. Torrea, Weighted norm inequalities for commutators of strongly singular integrals, Indiana Univ. Math. J. 40 (1991), no. $4,1397-1420$.

[11] D. Gilbarg and N. S. Trudinger, Elliptic Partial Differential Equations of Second Order, Second edition, Grundlehren der Mathematischen Wissenschaften 224, SpringerVerlag, Berlin, 1983.

[12] V. S. Guliev, F. Deringoz and S. G. Gasanov, Commutators of a fractional maximal operator on Orlicz spaces, translation in Math. Notes 104 (2018), no. 3-4, 498-507.

[13] P. Hajłasz and J. Onninen, On boundedness of maximal functions in Sobolev spaces, Ann. Acad. Sci. Fenn. Math. 29 (2004), no. 1, 167-176.

[14] J. Hart, F. Liu and Q. Xue, Regularity and continuity of local multilinear maximal type operators, to appear in The Journal of Geometric Analysis. https://doi.org/10.1007/s12220-020-00400-7

[15] T. Heikkinen, J. Kinnunen, J. Korvenpää and H. Tuominen, Regularity of the local fractional maximal function, Ark. Mat. 53 (2015), no. 1, 127-154.

[16] J. Kinnunen, The Hardy-Littlewood maximal function of a Sobolev function, Israel J. Math. 100 (1997), 117-124.

[17] J. Kinnunen and P. Lindqvist, The derivative of the maximal function, J. Reine Angew. Math. 503 (1998), 161-167. 
[18] J. Kinnunen and O. Martio, Hardy's inequalities for Sobolev functions, Math. Res. Lett. 4 (1997), no. 4, 489-500.

[19] J. Kinnunen and E. Saksman, Regularity of the fractional maximal function, Bull. London Math. Soc. 35 (2003), no. 4, 529-535.

[20] S. Korry, Boundedness of Hardy-Littlewood maximal operator in the framework of Lizorkin-Triebel spaces, Rev. Mat. Complut. 15 (2002), no. 2, 401-416.

[21] _ A class of bounded operators on Sobolev spaces, Arch. Math. (Basel) 82 (2004), no. 1, 40-50.

[22] F. Liu, Continuity and approximate differentiability of multisublinear fractional maximal functions, Math. Inequal. Appl. 21 (2018), no. 1, 25-40.

[23] F. Liu and $\mathrm{H}$. Wu, On the regularity of the multisublinear maximal functions, Canad. Math. Bull. 58 (2015), no. 4, 808-817.

[24] - On the regularity of maximal operators supported by submanifolds, J. Math. Anal. Appl. 453 (2017), no. 1, 144-158.

[25] F. Liu and S. Xi, Sobolev regularity for commutators of the fractional maximal functions, Banach J. Math. Anal. 15 (2021), no. 1, Paper No. 5, 36 pp.

[26] F. Liu, Q. Xue and P. Zhang, Regularity and continuity of commutators of the HardyLittlewood maximal function, Math. Nachr. 293 (2020), no. 3, 491-509.

[27] H. Luiro, Continuity of the maximal operator in Sobolev spaces, Proc. Amer. Math. Soc. 135 (2007), no. 1, 243-251.

[28] - On the regularity of the Hardy-Littlewood maximal operator on subdomains of $\mathbb{R}^{n}$, Proc. Edinb. Math. Soc. (2) 53 (2010), no. 1, 211-237.

[29] (2018), no. 1, 147-161.

[30] M. Milman and T. Schonbek, Second order estimates in interpolation theory and applications, Proc. Amer. Math. Soc. 110 (1990), no. 4, 961-969.

[31] P. Zhang, Characterization of Lipschitz spaces via commutators of the HardyLittlewood maximal function, C. R. Math. Acad. Sci. Paris 355 (2017), no. 3, 336-344.

[32] P. Zhang and J. Wu, Commutators of the fractional maximal function on variable exponent Lebesgue spaces, Czechoslovak Math. J. 64 (2014), no. 1, 183-197. 
Feng Liu and Guoru Wang

College of Mathematics and System Science, Shandong University of Science and Technology, Qingdao, Shandong 266590, China

E-mail addresses: FLiu@sdust.edu.cn, grwang2020@163.com 